Portland State University

PDXScholar

6-8-1994

\title{
Conversational Structure in Electronic Mail Exchanges
}

Linda I. Godson

Portland State University

Follow this and additional works at: https://pdxscholar.library.pdx.edu/open_access_etds

Part of the Bilingual, Multilingual, and Multicultural Education Commons Let us know how access to this document benefits you.

\section{Recommended Citation}

Godson, Linda I., "Conversational Structure in Electronic Mail Exchanges" (1994). Dissertations and Theses. Paper 4848.

https://doi.org/10.15760/etd.6724

This Thesis is brought to you for free and open access. It has been accepted for inclusion in Dissertations and Theses by an authorized administrator of PDXScholar. Please contact us if we can make this document more accessible: pdxscholar@pdx.edu. 


\section{THESIS APPROVAL}

The abstract and thesis of Linda I. Godson for the Master of Arts in TESOL were presented June 8, 1994, and accepted by the thesis committee and the department.

COMMITTEE APPROVALS:
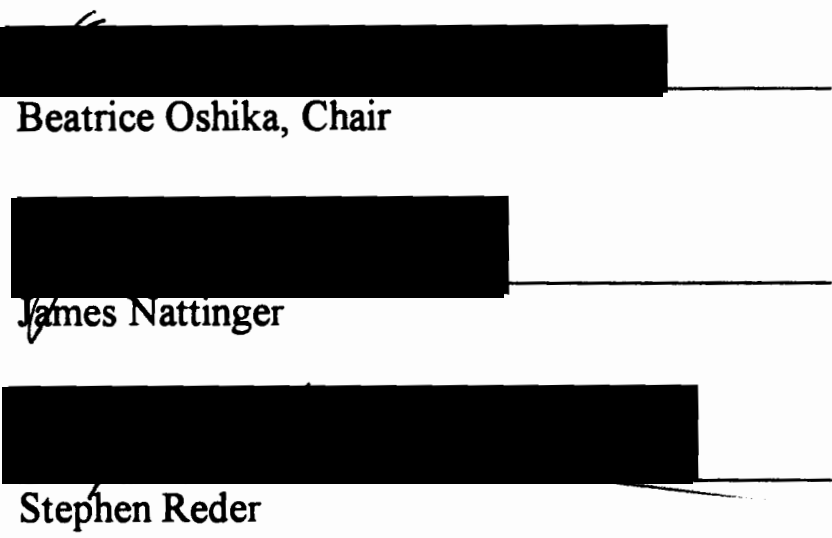

DEPARTMENT APPROVAL:

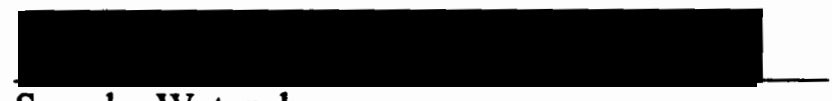

Suwako Watanabe

Representative of the Office of Graduate Studies

James Nattinger, Chair

Department of Applied Linguistics

ACCEPTED FOR PORTLAND STATE UNIVERSITY BY THE LIBRARY

by

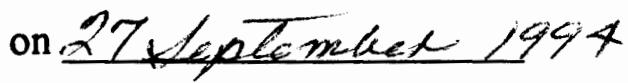




\begin{abstract}
An abstract of the thesis of Linda I. Godson for the Master of Arts in TESOL presented June 8, 1994.

Title: Conversational Structure in Electronic Mail Exchanges.

Electronic mail has become a widely used medium of communication in academia, government, and business. It is unique as a communication medium because it makes conversations across time, space, and organizational levels possible. The ability of electronic mail to "forward" a message allows for the creation of chains that preserve the entire conversation for each participant. This appears to be a new linguistic form in which the interactive features of spoken conversation are realized using electronically transmitted text.
\end{abstract}

The purpose of the present study was to determine the extent of the similarities and differences between spoken conversation and electronic mail exchanges. The research questions addressed were 1) What techniques that occur in spoken conversations also occur in electronic mail exchanges?, and 2) How are the techniques used in spoken conversations modified or different in electronic mail exchanges?

The data used in this study consisted of electronic mail text collected by the author in the course of her daily work in the data processing division of a large financial institution. The authors were computer technicians and middle managers with a wide diversity of educational backgrounds. Sixteen samples of message "chains" that contained at least three individual messages were selected for in-depth analysis. These samples were analyzed for conversational openings and closings, turn-taking mechanisms, adjacency pairs, and repetition. 
Of the structural features studied, repetition was used in ways most similar to its uses in spoken conversation. The feature having the most differences from spoken interaction was the turn-taking system. In the electronic mail exchanges there was more variation in the sender's selection of the next sender, including the option for multiple simultaneous replies. Openings and closings took many forms, some of them the same as in spoken conversation. Among the forms that differed were openings that resembled the salutation in a letter and closings that followed each individual message in a "chain." Adjacency pairs such as questions and closings were paired as in spoken conversation, while openings, thanks, and apologies occurred as single utterances. 
CONVERSATIONAL STRUCTURE

IN ELECTRONIC MAIL EXCHANGES

\author{
by \\ LINDA I. GODSON
}

A thesis submitted in partial fulfillment of the requirements for the degree of

\title{
MASTER OF ARTS \\ in \\ TESOL
}

Portland State University

1994 


\section{TABLE OF CONTENTS}

PAGE

\section{CHAPTER}

I INTRODUCTION

II SPOKEN CONVERSATION . . . . . . . . . . . . . . . . . . . . . 5

The Context of a Conversation $\ldots \ldots \ldots$

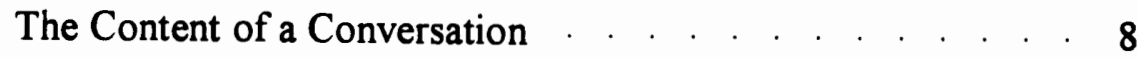

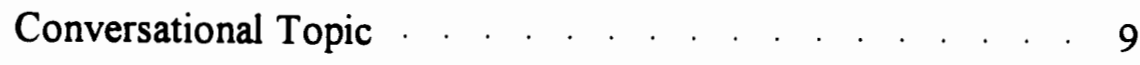

Functions of Conversation . . . . . . . . . . . . . . . 10

Coherence . . . . . . . . . . . . . . . . . . 12

The Structure of Conversation . . . . . . . . . . . . . . . 13

The Turn-taking System

Adjacency Pairs . . . . . . . . . . . . . . . . . . . . . . . . . . . . . 19

Repetition . . . . . . . . . . . . . . . . . . . . . 22

Conclusion . . . . . . . . . . . . . . . . . . . . . . . . 23

III RESEARCH DESIGN AND METHODOLOGY . . . . . . . . . 25

Research Design . . . . . . . . . . . . . . . . . . . 27

Data . . . . . . . . . . . . . . . . . . . . . . . . 28

Data Collection . . . . . . . . . . . . 31

Privacy and Confidentiality . . . . . . . . . . . . 31

Data Analysis . . . . . . . . . . . . . . . . . . . 32

IV ANALYSIS OF ELECTRONIC MAIL CONVERSATIONS . . . . 34

Conversational Openings and Closings . . . . . . . . . . 35

The Turn-taking System . . . . . . . . . . . . . . . . 41

Adjacency Pairs . . . . . . . . . . . . . . . . . . . 49 
Repetition . . . . . . . . . . . . . . . . . . . . . 54

V RESULTS . . . . . . . . . . . . . . . . . . . . . . . . 62

Conversational Openings and Closings . . . . . . . . . . . . . 62

The Turn-taking System . . . . . . . . . . . . . . . . 63

Adjacency Pairs . . . . . . . . . . . . . . . . . . . . . . 65

Repetition . . . . . . . . . . . . . . . . . . . 66

Summary . . . . . . . . . . . . . . . . . . . . . . 67

VI CONCLUSIONS AND DIRECTIONS FOR FUTURE RESEARCH 68

Conclusions . . . . . . . . . . . . . . . . . . . . . . . 68

Applications. . . . . . . . . . . . . . . . . . . . . 70

Directions for Future Research. . . . . . . . . . . . . . 71

REFERENCES . . . . . . . . . . . . . . . . . . . . . . . . . . . 74

APPENDIX: ELECTRONIC MAIL CORPUS . . . . . . . . . . . . . . . . 78 


\section{CHAPTER I}

\section{INTRODUCTION}

Over the last decade electronic mail has become a widely used medium of communication in academia, government, and business. The sender types a message on a video screen or teletype, specifies an addressee, and a computer manages its transmission, delivery, and storage. It is unique as a communication medium because it makes conversations across time, space, and organizational levels possible. Relying primarily or, in some systems, wholly on text, it is different from other electronic messaging systems such as telephone and facsimile. The medium is writing, but ongoing interaction with small time lapses between turns resembles spoken conversations. Thus electronic mail shares features with both speaking and writing. Further, there is flexibility to send messages simultaneously to multiple recipients, to forward messages, and to create conversational "chains" of messages. The history of a conversation can be accumulated and shared with new participants. These features encourage the establishment of ties between users who normally would not communicate (Feldman, 1987).

The power and popularity of electronic mail were unexpected in many of the organizations now using it. According to Sproull and Kiesler (1991), when the ARPANET was set up to allow scientists to have access to computers at other universities, the electronic mail feature became surprisingly popular. Other systems have been similarly successful even though within an organization there are wide differences

among individuals in the use of electronic mail (Reder \& Schwab, 1989). Those who rely on it for both work and personal communication may have equipment at home as well as at their work site (Porter, 1984). 
In the popular new computer networking magazine, Wired, Paul Saffo (1994) observes that even though the media choices available to us continue to grow, text remains the central vehicle for electronic communication.

In fact, the written word doesn't just remain; it is flourishing like kudzu vines at the boundaries of the digital revolution. The explosion of e-mail traffic on the Internet represents the largest boom in letter writing since the 18th century. Today's cuttingedge infonauts are flooding cyberspace with gigabyte upon gigabyte of ASCII musings. (America Online, 3-13-94)

Fish, Kraut, and Chalfonte (1990) assert that informal communication is of crucial importance in the workplace. With electronic means this can be accomplished without the requirement of physical proximity. Neither is there a requirement of temporal proximity since time lapses between creation and sending, and sending and receiving are elastic. Sproull and Kiesler (1986) have pointed out that gender and organizational status information may be lacking. In a study based on self-reports by senders and receivers, they concluded that electronic messaging behavior tends to be undifferentiated by status. It also tends to be uninhibited and non-conforming.

My interest in electronic mail conversations is a direct result of my job in the Information Services Division of a large corporation. As a project manager and operability consultant, I use electronic mail for at least half of my communication with my colleagues. Most of this communication is in the form of extended conversations with multiple individuals. Since most of my colleagues also rely heavily on electronic mail to do their work, it has become crucial to our organization. A mail system outage is handled with the same procedures as a critical financial system failure.

In addition to the functions of exchanging information and maintaining social ties, researchers have identified some less obvious uses for electronic mail. Sproull and Kiesler (1991) describe the "Does anybody know?" function as one in which someone can send an inquiry to a large number of recipients with a high probability of getting a useful reply. 
The sender is willing to admit ignorance and the recipient is likely to reply because it is easy to do and because of what the authors call "electronic altruism."

In my organization, a large financial institution, electronic mail is seen as a potentially powerful political weapon. This is usually wielded in the form of a seemingly innocent copy of a message sent to an important manager. This can be tricky because there are also rules about sending mail up and down the organizational hierarchy (contrary to the findings of Sproull and Kiesler cited above). These rules extend to when it is acceptable to ignore a message. Just as in face-to-face conversation, the absence of a reply can send a strong message.

These observations do not, however, exhaust the possible lines of inquiry into electronic mail. An entirely new linguistic form, according to Reder and Schwab (1989), has been created. They refer specifically to the chain of messages generated with multiple uses of the "forward" function. All past exchanges are present at every point in the conversation, and there may be any number of participants. Murray (1991) included this form of electronic mail in her analysis of the communication channels used by an IBM project leader. The focus of her study was how the project members used the range of different media resources available to them. Blackman (1990) touches on this phenomenon in his analysis of bulletin board systems. Although some of his observations may apply to electronic mail, they differ in a crucial way. All messages are sent to the central bulletin board and all messages are available for replies. This lack of specific addressees creates a different, perhaps also new, form of communication.

The fact that individuals may choose an electronic mail conversation as an alternative to a face-to-face conversation suggests that the two media may have similar functions. In addition, since electronic mail is too new to have developed rigid conventions for its use, it may be that it has borrowed some of the features of spoken conversation. It is reasonable to expect, therefore, that some of the techniques of spoken 
conversation will be found in electronic mail "chains." One may also expect that where tools available in face-to-face interaction, such as eye contact and body language, are absent, some substitutes will be introduced. In order to determine what these similarities and adaptations are it will be necessary to review the research on spoken conversation that has been done by anthropologists, sociologists, and linguists. 


\section{CHAPTER II}

\section{SPOKEN CONVERSATION}

Spontaneous talk among two or more individuals is the primary site for language use (Ford, 1993). Most human activity involves conversation in which people plan, debate, discuss, gossip, and carry out their social processes (Clark, 1989). For children, interaction with speakers of their native language is fundamental to the process of language development and understanding.

Within the larger domain of discourse, or sequences of either spoken or written utterances, linguists understand conversation as a form of discourse that involves at least two participants who together construct a meaningful whole as a result of their spoken interaction. Clark (1989) defines a conversational discourse as "a sequence of utterances produced as the participants proceed turn by turn" (p. 259). He goes on, however, to point out that conversations are highly coordinated activities in which both speaker and listeners work to ensure mutual understanding. This view of conversation as a shared activity is basic to the work of conversational analysis.

Conversational analysis is concerned with analyzing actual interactions occurring in their natural environments. This empirical analysis aims at developing a descriptive grammar of natural conversation. Included in such a grammar are the functions that can be accomplished by the conversation, the content and how it is determined, and the structure that supports both the function and the content. Such abstract analysis must also take into account local idiosyncratic conversational environments and determine the fit between general principles and specific actualization (Schenkein, 1978). An example from 
Wardhaugh $(1985$, p. 155) illustrates a simple structural pattern with a subtle meaning that is conditioned by the situation.

Wife: Are you going to walk Rufus?

Husband: Did the bloke come about the TV yet?

Wife: No.

Husband: He will have to wait then.

The wife's first question, normally requiring an answer, elicits another question.

However, if we consider the husband's question to be the first part of a pair consisting of question and answer, we can see that the first and last utterances are also a question and answer pair. This adequately describes the structure of the conversation, but there is more going on than requests for information. The husband has refused to walk the dog, but he has done it indirectly. He shows his irritation at the request in his final terse statement. Our understanding of these subtleties has to do with the particular relationship between the participants and with the kind of language used in this specific case

\section{The Context of a Conversation}

Understanding a conversation involves more than careful attention to the conversational data itself. One must also understand who the participants are, what information and background they share, events surrounding the conversation, the physical setting, and the location in time. Some of this information is independent of the conversation, but more often it influences and is reflected in the conversation. (Wardhaugh, 1985)

The participants may be friends, colleagues, or strangers. The degree of involvement among participants will be a function of these relationships, and is somewhere on a continuum between detachment and commitment (Wardhaugh, 1985). There are interesting consequences if the participants assume different levels of involvement. 
According to Tannen (1990), this is frequently related to gender differences. Depending on the relationships among the participants, each will make an effort at establishing credibility. They may accomplish this by using a shared vocabulary or introducing group-specific jargon (Wardhaugh, 1983). The participants may also be aware of an audience that is not part of the conversation but that imposes constraints on it.

The individual personalities of the participants may be shared knowledge at the beginning of the conversation, revealed in the course of it, or a combination of both. In any of these cases, each member of the group will display her own personality and evaluate the other individuals in many ways. Among the tools participants may use to project their personalities are exaggeration, posturing, tentativeness, skepticism, oversimplification, allusions, and metaphor (Wardhaugh, 1983). These can relate to the "face" that the speaker and listeners present. Goffman (1955) defines "face" as "the positive social value a person claims for himself in social contact situation with a pattern of verbal and non-verbal acts" (p. 319). There is a strong bias to maintain positive face and to avoid negative face. The participants do this both for themselves and for the other participants. The techniques available for "saving face" include ceremonial treatment, avoiding facts that embarrass, circumlocutions, ambiguity, hints, innuendo, and wellplaced pauses. If these techniques fail so that loss of face occurs, there is a mechanism for correcting it. According to Goffman, there are four steps that characterize this form of interpersonal ritual behavior: challenge, offering, acceptance, and thanks. Loss of face may also be corrected by using jokes, suggesting that the offender was not himself, or by offering an apology.

Conversations happen in a situational context that involves previous events, the current environment, and expected future events. Previous events may include other conversations or shared experiences. The current environment includes the physical setting such as a home, office, or public place. If the owner of the location is a participant 
this will affect her relationship to the other participants. Location can have a strong influence on the degree of formality, and the number of formal ritual expressions used in the conversation (Wardhaugh, 1983). Expected future events may be unspoken shared knowledge or explicitly referred to in the conversation.

The location of a conversation in an external time frame also constrains its form and content. Time adverbs at the beginning or end of an utterance such as "yesterday" or "next year" depend on the external time frame for interpretation. Other temporal clauses introduce new time frames, change an existing time frame, or move between specific and generic time frames (Ford, 1993). For example, "When I graduate in June" establishes a new time frame, whereas "Graduation time is exciting" moves the previous specific time to a generic one.

\section{The Content of a Conversation}

Although we commonly think of a spoken conversation as a topic-related collection of utterances that the participants exchange, there are other important components. These are meta-conversational utterances and non-verbal communication. Metaconversational utterances provide a frame for other utterances by defining their intent or meaning. For example, "Was that an order?" asks for a definition of an utterance; "Was that a joke?" asks about intent. (Wardhaugh, 1983) Non-verbal communication, according to Wardhaugh (1983), takes precedence over spoken communication when they oppose each other. It takes many forms on a continuum from explicit to subtle and from conscious to automatic. At one extreme is sticking out one's tongue to indicate distaste, at the other is a quick glance at the clock to indicate boredom. Both of these can reinforce meaning and provide feedback. Emotion can be indicated with unconscious postures, facial expression, laughter, or tears. Empathy can be established by mirroring body 
language and with eye contact. Emotional distance can be communicated by limiting eye contact.

A very important form of non-verbal communication is the physical distance between participants. As the distance varies, the definition of the relationship between the participants varies accordingly. In research with educated professionals, including blind subjects, using observation, interview, and participant observation, Hall (1964) concluded that Americans have four distance groups. A single conversation may move among them. The groups are intimate at less than four feet, casual-personal at four to seven feet, social-consultative at seven to twelve feet, and public at greater than twelve feet. Speech style for each of these four groups is consistent with the relationships defined by the physical distance, with the casual-personal group using informal style and the social-consultative group using impersonal business style.

\section{Conversational Topic}

The interactive nature of conversation creates an environment in which topic selection is a shared enterprise. An agenda is not usually determined in advance or dictated by a single individual unless the conversation is part of a formal meeting or preplanned event. Rather, the participants negotiate topics in the natural course of the interaction. In this process they will need to determine what topics are acceptable, to present information as reasonable to be shared, to allow multiple contributions, and perhaps to ask for help to construct an agenda. Misunderstandings will also have to be corrected.

The participants decide what topics are acceptable partly by using their shared knowledge of taboo subjects and individual sensitivities. They may understand a topic to be unacceptable by what is not said. On the other hand, a topic can be explicitly rejected with a comment like "What a terrible thing to say!" To allow shared construction of a 
topic they can use vague expressions like "something like," "that kind of thing," "more or less," and "roughly" (Wardhaugh, 1985). A participant may offer information as reasonable to be shared by introducing it with an "if" clause such as "If you like scary movies. ..." Misunderstandings can be avoided or corrected by checking ("Did you really mean that?"), backtracking ("But I thought you said. . ."), and hypothesizing ("If you were talking about your sister. .") (Wardhaugh, 1983; Ford, 1993).

A speaker can introduce a topic change by saying, "That reminds me of," or "Speaking about," and then continuing with the new topic. If she wishes to revive a previous topic she can say something like, "As I said earlier," or "Going back to X" (Wardhaugh, 1983, p. 143). A third possibility for introducing a topic is spontaneous awareness of something in the envirnoment signalled by, "Did you see that?" Variations of this gambit are commonly used to initiate conversations with strangers.

\section{Functions of Conversation}

People use conversations to accomplish a large number of functions including sharing information, planning for action, mutual support, entertainment, and problem solving. Conversation may also be used to perform acts with words such as promising, threatening, insulting, challenging, or refusing (Labov, 1972). In addition, most conversations have components that contribute to social solidarity. The concern to maintain or enhance relationships is expressed in phatic language, defined by Malinowski (1923) as "language that fulfills a function to which the meaning of its words is almost completely irrelevant" (p. 149). Examples are ritualistic greeting routines, inquiries about health or the weather, statements of the obvious, stylized apologies, and expressions of gratitude.

Maintaining cooperation is central to all the functions of conversation because without it there would be no shared meaning or action. Cooperation entails keeping the 
conversation going, contributing appropriately, and avoiding confrontation. Keeping the conversation going requires that the listeners maintain awareness and understanding of the speaker, but it also may involve unconscious mimicry of accent, language style, lexical choices, and grammatical structures (Wardhaugh, 1983). Contributing appropriately requires that the participants acknowledge what the others say, allow the others a full hearing, and contribute in such a way that the conversation moves forward. Avoiding confrontation can be accomplished by using euphemisms or understatement, and by giving the others the benefit of the doubt. Wardhaugh (1983) points out that a participant can opt out of cooperation, and thereby remove herself from the conversation, by silence, abusive words, using taboo words or gestures, or putting others down.

Grice (1975) labeled the underlying mechanism that makes productive conversation possible the Cooperative Principle. He divided the requirements for cooperative talk into four categories: quantity, quality, relation, and manner. Included in the category of quantity is the requirement that contributions be as informative as needed for the purposes at the time, but not more so. Quality relates to the assumption that the participants will tell the truth, and will not assert that for which they lack adequate evidence. The category of relation covers the requirement to be relevant. Manner includes the expectation that speakers will avoid obscurity, avoid ambiguity, be brief, and be orderly. These maxims, as Grice calls them, are learned in childhood and are unconsciously assumed in normal human interactions.

All of us, however, have experienced conversations in which at least some of these maxims are not followed. Grice (1975) describes four ways that this may happen. A participant may quietly violate a maxim and run the risk of misleading the others. She may opt out of the conversation by asserting that she has nothing further to say. More interesting is the situation in which two of the maxims clash. This can happen if the participant lacks adequate evidence for information that is required. Finally, she may 
blatantly fail to fulfill a maxim, which Grice calls flouting. That is, she chooses to violate a maxim with a specific purpose in mind. In such a situation the hearer first must determine if she is merely being misleading, or is opting out, or experiencing a clash. If none of these apply, the assumption is that the speaker is using the mechanism of flouting a maxim to achieve a communicative function. Grice calls this "conversational implicature." What happens is that the hearer is aware that a maxim is violated at the level of what is said, but assumes that the Cooperative Principle continues to be observed at the meaning level. Grice gives the example of a testimonial about a pupil who is a job candidate that says: "Dear Sir, Mr. X's command of English is excellent, and his attendance at tutorials has been regular. Yours, etc." The recipient of this letter has to assume that the writer is not opting out since he answered the request. Rather, the minimal information in the letter implies that the writer knows more, but is unwilling to write it because it is unfavorable.

\section{Coherence}

Coherence, according to the Longman Dictionary of Applied Linguistics, (1985, p. 45), is "the relationships which link the meanings of utterances in a discourse or of the sentences in a text." In conversation coherence is achieved through cooperative action. The participants create it as the conversation develops. It is emergent in time, and undergoes transformations as the situation or topic evolves (Hopper, 1983). According to Sanders (1983) coherence is the result of meaning relations and structural relations. To identify meaning relations the participants must identify which of multiple topics is being addressed, mark topic boundaries, and identify the meaning of what is communicated about the topic. Sanders observes that the participants use primarily verbal means to accomplish the tasks related to determining meaning. They determine communicative value either semantically and literally, or in terms of implicature, or as illocutionary acts 
such as ridicule. Structural relations, on the other hand, are more likely to be non-verbally marked. Grammatical markers within sentences are intonational, using vocal stress and tonal duration. At the discourse level the markers may include intonation, but also can be indicated by respiration rate, eye contact, body position, or a change in physical distance from other participants.

\section{The Structure of Conversation}

It is convenient to look at the organizational structure of a conversation as a speech event as having three major parts: the opening, the body, and the closing. Each part has a particular function and an appropriate structure. Although there may be formulaic components throughout a conversation, they most usually occur at the beginning and at the end. Such ritual exchanges exist on a "scale of fixity" so that variation can be introduced without obscuring the ritual intent of the utterance. Tannen (1989) claims that in American English formulas are not rigid. For example, the ritual farewell to a person leaving on a journey might be "Have a good trip," or "Have a nice trip," or even "Have a great trip" (p. 39). All of these mean the same thing.

To open a conversation it may be necessary to create the possibility of initiating a conversation. Wardhaugh (1983) refers to these as "pre-openings" (p. 124). Possibilities for a pre-opening are a smile, looking at an object, or eye contact. Following the preopening, the speaker must initiate talk in spite of the social inhibitions against interrupting, breaking a silence, or talking to strangers. These inhibitions explain why some ritual openings are apologies like "Excuse me" and "Sorry to bother you, but." Another possible opening is a question about an impersonal subject like the time. For people who already know each other, the most common opening is either "Hi" or "Good morning (or afternoon or evening)." In a study of conversational openings in a Boston business environment, Brown and Ford (1961) found that "Hi" was more common to 
intimates and subordinates while "Good morning" was used for distant acquaintances and superiors. The use of first or last names and titles in an opening can further serve to define status or difference in age.

Another function of conversational openings is to establish a bond between the participants. A topic of mutual concern like the weather or the immediate surroundings can be used for this purpose, as can a request for information like "Where do you live?" (Wardhaugh, 1983). Another possibility is an introduction like "I'm Mary," to which the listener can naturally reply, "I'm John."

The body of a conversation is, as already noted, a cooperative activity in which all the participants construct a coherent whole. They are responsible for keeping the conversation going, for connecting their individual utterances to previous ones, for getting the message right, and for clarifying the structure or direction of the conversation. They have many tools available to do this. Feedback such as "And then?" or "Exactly" serves to allow the speaker to continue, while connectors such as "if," "but," and "so" can provide continuity with previous utterances. Confirmation checks and understanding checks can be used to be sure the meaning gets across (Wardhaugh, 1983).

Digressions that are outside the current topic may be inserted into a conversation. These "side sequences" allow the participants to express unrelated thoughts before they are forgotten, but do not permanently change the topic. The beginning of a side sequence may be indicated verbally with "by the way," or "incidentally," or non-verbally with a change in intonation or body position. A return to the original conversation can be marked with something like "well, anyway" or a return to the original voice tone and posture. (Hatch, 1992)

The interactive language used in spoken conversation is constrained by the limited time available for the participants to plan their contributions, the restrictions of human short term memory, and the unpredictable and fluid organization of the conversation as a 
whole. Such language tends to be fragmentary, consisting primarily of single words, phrases, and clauses. In addition, spoken word order must not require that long word strings be stored before meaning can be assigned. For example, in speech the object most often immediately follows the verb, but in writing it can be postposed. The sentence, "John addressed a series of appeals to the crowd," is normal for speech, but when the object is moved we get "John addressed to the crowd a series of appeals." This latter is not acceptable spoken English. (Allerton, 1991)

In order to facilitate quick processing of the information in an utterance, a speaker may use a topic-comment structure in which the topic is "left dislocated." This structure is useful for introducing a new topic and for beginning a side sequence. The following are two examples of topic-comment structure cited by Hatch (1992) from counseling interviews:

C. Uh, about money, uh he has a darn good job and makes good money.

C. John he's like about twice my age. (p. 239)

Another feature of interactive talk is the absence of explicit cohesive ties and syntactic structures that might be necessary in written language. Instead, utterances are connected by virture of their sequence in time, or "nextness" (Hatch, p. 240). For example,

C: As I said + I can't discuss-very few things can I discuss with him + + "I don't want to talk about it" + he walks outa the room.

In written discourse, this might be:

There are very few things I can discuss with him. When I try, he says that he doesn't want to talk about it and leaves the room. (Hatch, p. 240)

The structures, "there are," "when I try," and "and," that are present in the written version are not needed in the spoken utterance because the speakers are already focused on the topic and assume that the clauses are connected because they occur in sequence. They also have available to them intonation and body language as indicators. 
The functional differences between speech and writing also have linguistic indicators. Monitoring of the communication channel with fillers like "well," "I mean," and "you know," are significant in speech only. Emphatic particles likewise mark spoken discourse. Vagueness and hedges such as "and so on," and "sort of," though used in writing are more common in speech. (Chafe, 1982)

Participants can close a conversation explicitly, with a ritual ending such as "Got to run," or "Well, back to work," or implicitly with body language. Diminished eye contact, standing up, and glancing at a clock, all indicate that the conversation is about to conclude. Pre-closings such as "I just remembered," allow the other participants to make their final contributions. Not all conversations end amicably, however. A rude closing such as silence and walking away can serve to end an acrimonious conversation or argument. (Wardhaugh, 1983)

\section{The Turn-taking System}

The most obvious structural feature of spoken conversation is turn-taking. Sacks, Schegloff, and Jefferson (1974) describe the system involved in terms of "turnconstructional units." These are units of talk that are the minimal content of a single turn, and which may be made up of a single word, a phrase, a clause, a sentence, or multiples and combinations of any of these. The constraints on what may be included in a turnconstructional unit are external to the turn-taking system. These include the Cooperative Principle (Grice, 1975), external time constraints, and local social conventions. Although the content of a single unit is highly variable, it is common for it to have three parts: a reference to the previous unit, a new part for the current unit, and a part that relates to the succeeding unit.

A turn-constructional unit must have a completion point, or "transition-relevance place" so that the next speaker may begin her turn (Sacks, Schegloff, \& Jefferson, 1974, 
p. 13). The completion point is usually projectable before it occurs. This allows the next speaker to prepare her contribution and to begin speaking at the appropriate time. For example, if the unit is a question like "What time does the show start?" the first word indicates that the turn will end at the question mark.

The conversational system made up of turn-constructional units that end with transition-relevance places has the following characteristics: (Sacks, Schegloff, \& Jefferson, 1974)

1. Speaker change recurs. This happens because at a turn-transitional place the speaker either selects the next speaker, the next speaker self-selects, or the speaker continues. This is a precedence order so that no one can self-select if the speaker selects the next speaker. The speaker may continue only if no one self-selects. The turn markers are predominantly non-verbal using stress, tonal duration, loudness, and pitch change. Speaker selection devices include eye contact and tag questions such as "don't you agree?" or "you know." Self-selection techniques include beginnings such as "well," "but," and "so."

2. One party talks at a time. Overlap at transition-relevance places is common, but brief. Transitions from one turn to the next without a gap or overlap are common. Most others have only a slight gap or overlap. This is because self-selection requires that there be no gap. If there is, the speaker may continue, or someone else may self-select.

3. Turn order varies. This is a consequence of the allocation rules. The combination of a series of options for determining the next speaker and independent operation at each transition-relevance place necessarily results in variation of turn order. In other words, control of turn-taking order is local, operating turn by turn. However, factors such as the need to accommodate problems of understanding and the need for clarification may bias the rules in the direction of the next speaker being the previous speaker. Given this restriction, it may also be the case that there is a preference for two-person conversations. 
4. Turn size is not fixed. A single turn-constructional unit may be made up of any number and combination of single words, phrases, clauses, or sentences. There is pressure toward short units because potential next speakers will try to self-select at what they recognize as a turn-transitional place. On the other hand, the turn-taking option for the speaker to continue at a transition-relevance place contributes to creating longer units. This choice has the lowest priority, so it will not occur consistently, but when it does happen the turn size becomes larger. The choice to continue, moreover, may occur multiple times in a single turn.

5. The length of a conversation is not fixed or specified in advance. Since the next speaker is determined independently at each turn, the number of previous turns has no influence on how many turns may make up the conversation. The rules allow any nonspeaker to be next with no restriction on how many turns each speaker may have.

6. The number of parties can change. The selection rules are independent of the size of the group, but as the size of the group increases, there is bias toward reducing the length of a single turn. This is because there are more speakers attempting to self-select at the earliest opportunity. Also, when the group reaches four parties, the conversation may split in two. To avoid a split, there is a bias toward distributing turns across the entire group.

7. There are repair mechanisms for correcting turn-taking errors and violations. These mechanisms are organized with the same rules as turn-taking. If a speaker selects before a turn-transitional place has been reached, resulting in two parties talking at once, the original speaker will continue and the other will stop. If the speaker intends to select the next speaker but is not clear about the selection, another party may attempt a correction by saying, "Who me?"

The mechanisms available to the participants to accomplish turn-taking include both verbal and non-verbal activities. The linguistic devices are frequently ritualized. To keep 
the floor the speaker may use linking devices like "For example," "Take X," "The point is," or simply "aahh." To relinquish the floor without choosing the next speaker she may use a cliched ending such as "That's life," or Win some, lose some." To offer the next turn to a specific person she can use a combination of eye contact and "What do you think?" A participant may indicate that she is about to self-select for a turn by quick breath intake and body tension. Her opening might be, "But I thought." To interrupt explicitly, without waiting for a turn-transitional place, a speaker can say, "Excuse me," or, less politely, "Wait just a minute, that's wrong." (Wardhaugh, 1983, pp. 143-152)

\section{Adjacency Pairs}

Another way to look at the structure of a conversation is to see it as a sequence of paired exchanges, or adjacency pairs. Ford (1993) defines an adjacency pair as two conversational turns in which the first turn makes the second relevant. Schegloff (1972) points out, for example, that the status of an utterance as an answer rather than as an assertion depends on the existence of a preceding question. Other possibilities for the second half of a pair that begins with a question are another question, if clarification is needed, or silence that prompts the speaker to repeat the question.

Greetings are typically adjacency pairs such as "How are you?" followed by "Fine," or "Good morning" with a second "Good morning" as the response. Closings are similarly paired, with repetition a common form.

Another class of adjacency pairs includes proposals, offers, and invitations. Each of these will normally be followed by either acceptance or rejection (Clark \& Schaefer, 1989). Other types of pairs that are followed by acceptance or rejection are challenges, threats, and requests for action (Wardhaugh, 1983). Labov (1972) observes that a refusal to a request for action may be in the form of a question:
A. Shut up please! 
B. ..'ey, you tellin' me? (p. 125)

Thanks may also be followed by acceptance or rejection as when the respondent says either "You're welcome" or "It was nothing." Apologies can be similarly accepted or rejected (Clark \& Schaefer, 1989).

A request for information may be followed by acceptance, with the requisite information, or refusal. However, Labov (1972) points out that if the request is not followed by the information, and the discourse is coherent, it implies that there is shared knowledge that makes the reply make sense. His example is:
A. Are you going to work tomorrow?
B. I'm on jury duty. (p. 122)

The shared knowledge that makes B's assertion a reasonable reply to A's question is that it is not possible to go to work when one is on jury duty. A request for information may also be followed by a request for clarification in the form of a question. In this case a second adjacency pair would be embedded between the two parts of the first pair.

A compliment may be followed by either agreement, disagreement, acceptance, or rejection. Pomerantz (1978), in a study of naturalistic conversations, showed that there is a preference for agreement over disagreement, but that the requirement to minimize selfpraise causes a preference for rejection over acceptance. These conflicting requirements result in a wide variety of responses to compliments. When the compliment is indirect, agreement is more likely. If it is direct and the recipient wants to accept it she may downgrade the compliment. Another solution to the dilemma is a referent shift in which the compliment is shifted to another person. Another graceful solution is to respond with another compliment so that when A compliments B, B then compliments A.

Assertions require a response that indicates evidence of understanding. This can be in the form of continued attention, initiation of a relevant next contribution, acknowledgment ("yeah," "uh huh"), or repetition of the assertion either verbatim or 
using a paraphrase. The response, in turn, requires acknowledgment so that the process is recursive. A discussion about the identifying number on a book shows this:

A. F six two.

B. F six two.

A. Yes

B. Thanks very much.

B's repetition of the number shows understanding and A's acknowledgment in turn confirms it. (Clark \& Schaefer, 1989, p. 268)

Schegloff (1972) discusses another recursive pattern that occurs in adjacency pairs. In what he calls an "insertion sequence" a question and answer sequence is inserted between an initial question and its final answer. This can be repeated multiple times in the same conversational sequence. His invented example (with clarifying indentation added) is:

A. Are you coming tonight?

B. Can I bring a guest?

A. Male or female?

B. What difference does that make?

A. An issue of balance

B. Female

A. Sure

B. I'll be there. (pp. 78-79)

The three levels here would appear to be comprehensible by the participants because each utterance is brief. Because the participants must hold the initial question and all the intervening information in short term memory, it is doubtful that many more levels, or extended utterances within three levels, would be possible in naturally occurring conversations.

The cooperative work of maintaining a coherent conversation sometimes requires that the hearer confirm what the speaker said or that the speaker check with the hearer that she was understood. These confirmation checks and understanding checks are another 
form of an adjacency pair. Labov (1972) points out a variation on this in which the speaker requests confirmation of something other than the initial assertion:

A. Oh, so she told you.

B. Yes. (p. 124)

The pairing requirement in conversations is so strong that when it is ignored the silence itself has meaning. If a greeting elicits no response, and the speaker believes she was heard, she will probably assume that the hearer is hostile or arrogant. The same applies to an ignored question or request. Silence following a challenge or a threat is equally unacceptable. (Wardhaugh, 1983) The exceptions to the pairing requirement include rhetorical questions and riddles, although a riddle may elicit an acknowledgment (Schegloff, 1972). In these cases the speaker is introducing a subsequent assertion or punch line.

\section{Repetition}

According to Tannen (1989) repetition of sounds, words, phrases, clauses, and utterances serves several functions in spoken conversation. It is particularly useful because of the dynamic nature of conversation that requires the participants to plan their contributions while either speaking or listening. Repetition enables the speaker to produce fluent speech while formulating what to say next. She can repeat a question or paraphrase an assertion, for example. By doing so the speaker also facilitates comprehension since the listeners may not have heard the entire utterance. Repetition can also be used to link the parts of a conversation by referring to previous utterances. As in other genres, foregrounding and emphasis can be accomplished with repetition. The utterance, "And he knows Spanish, and he knows French, and he knows English, ..." (p. 50), emphasizes that "he" knows many languages. At the same time it allows the speaker to extend the length of her utterance and to provide her listeners with an aid to 
comprehension. Further, episodes within a larger conversation can be bounded by repeating the beginning as a conclusion. Less cooperative, but also possible, is the use of repetition to mock, ridicule, or trivialize. Tone of voice plays a large part in communicating such intent.

Tannen (1989) also considers repetition to be a powerful tool for social maintenance. By echoing an utterance, perhaps with a few words changed, the speaker can create rapport while contributing to the cohesion of the whole. Some listeners do "shadowing," which is repeating the speaker with a small delay so the utterances overlap. This communicates agreement and support so long as it does not become irritating by being overdone.

There is a spectrum of repetition from an exact echo to a rough paraphrase. There may be a kernel phrase around which a conversation is structured that recurs as the topic develops. Structurally, repetition can be self-repetition as in "Do you want to split it? Do you want to split a Tab?" or allo-repetition as in "Do you want to split my Tab?" "No, I don't want to split your Tab." (Tannen, 1989, p. 54). In the former example the repetition modifies the original by changing a pronoun to a proper noun; in the second, a first person possessive is changed to second person. Tannen also gives an example of repetition used to create humor.

A. Rover is being so good.

B. I know.

A. He's being hungry. (p. 63)

The humor is created because a common construction, "being so good," is converted to an odd one, "being hungry."

\section{Conclusion}

Analysis of spoken conversation is a multi-dimensional undertaking. It must consider the context of the conversation, the explicit and implicit content including non- 
verbal components, the topics and how they are chosen and changed, the functions of the conversation, and the many levels at which it is structured. Of particular interest to the linguist are the markers that indicate initiation and termination of the conversation itself, the techniques for selection of a specific topic and topic change, and the mechanisms of the turn-taking system. Within these larger concerns, adjacency pairs are another structural unit that can help in analyzing a conversation. At the level of an individual utterance, there are linguistic structures characteristic of unplanned language that are the result of the nature of conversation itself.

The cooperative nature of conversation underlies all analysis since the participants construct the conversation as a joint enterprise. Topic selection, turn-taking, keeping the conversation going, maintaining positive "face" for the members of the group, and correcting misunderstandings all require that the participants listen attentively and contribute appropriately. The finely-tuned system that they use to accomplish such a complex task offers a challenging opportunity for the conversational analyst. 


\section{CHAPTER III}

\section{RESEARCH DESIGN AND METHODOLOGY}

Electronic conversations have both augmented and, in some cases, replaced spoken conversation. When the participants are separated by time, distance, or status, electronic mail may be the medium of choice for conducting business, arranging social events, or maintaining relationships. As this new medium is used to accomplish functions previously handled in face-to-face interaction, it is developing its own discourse structure. It is reasonable to assume that some of this structure is being transferred from the conventions of spoken conversation. At the same time, we can expect that those conventions will be modified to suit the limitations and opportunities of electronic conversations. It may even turn out that entirely new discourse structures and usages are emerging. Accordingly, this study has addressed these issues with two questions.

1. What techniques that occur in spoken conversations also occur in electronic mail exchanges?

The construction of the message "chains" that I collected for the study are like face-toface conversation in that multiple turns are taken by the participants. A closer examination shows, in addition, that some of their turn-taking mechanisms are similar. In this conversation fragment, Bill and Sarah are negotiating a time to meet to discuss their project. Bill has already told Sarah that he would like to talk to her.

Sarah: I assume the discussion is not an emergency - -Would you like me to set something up for next week?

Bill: How about this afternoon?

Sarah: $\quad 4: 30 ?$ 
Bill: $\quad$ Actually .... We could meet at 2:30 if you want?

Sarah: I prefer 4:30, if that's OK with you.

Bill: $\quad$ OK . . 4:30.

Although the medium allows Sarah to select Bill as the next speaker simply by sending him the first message, she uses a question that requires Bill to respond. Bill in turn responds with a suggestion in the form of another question so that Sarah must take the next turn. This questioning mechanism is common in spoken conversations. The only difference here is that the speaker does not use eye contact or body language to select the next speaker, but rather uses an electronic address.

We might also expect that repetition, used in spoken conversation to maintain coherence and to create rapport among the participants, will appear in electronic exchanges. In the conversation above, the meeting time, "4:30," appears three times. The function of this repetition appears to be a reinforcement of Bill's and Sarah's agreement to meet. Repetition may also appear in electronic conversations to compensate for the lack of non-verbal cues commonly used in spoken conversation.

2. How are the techniques used in spoken conversations modified or different in electronic mail exchanges?

In spoken conversation, a request for information may be followed by an acknowledgment of the request with some indication of when it might be satisfied. The requester then either accepts the response, perhaps with thanks, or rejects the response. In this electronic conversation, Henry is asking Sarah to supply information about a procedure he needs to use to change a computer process.

Henry: Sarah,

Can you tell me what is the procedure for having a new step added to the FRB grave shift runsheet?

Thanks

Henry 
Sarah: I am finding out who that should be and will let you know - When do you want the new procedure to start?

Henry: It can start as soon as I've done all the paperwork for it. Henry

Sarah: The procedure is to enter it into the Change System. You need to be sure that Operations is on the notification list. (I think you enter this - ) When the Ops supervisors get the change notification, they will update their run sheets.

In the first turn Henry not only makes his request, but thanks Sarah in advance for her response. This violates the convention in face-to-face interaction which would require that he wait for a response before expressing gratitude for it. He also redundantly "signs" his name. This suggests that he is using an electronic mail message more like a written letter than as part of a conversation. He repeats his name after his second message, but omits the thanks, presumably because he has already offered it. This may also explain why he does not respond to Sarah's final reply that contains the information he requested.

Modifications to the turn-taking system used in spoken conversation might also be required by differences introduced by the electronic medium. For example, an electronic mail message may be sent to multiple addressees without an indication of whether one, some, or all are expected to reply. In addition, the sender has the option of asking for a reply from anyone who can satisfy her request, or asking for a reply only if one of the addressees disagrees with her message. These options, not normally used in spoken conversation, may be expected to introduce difficulties for participants who assume the turn-taking system that applies in spoken conversation.

\section{$\underline{\text { Research Design }}$}

The approach I chose for this study was an in-depth ethnographic analysis of a small corpus of electronic mail. This is an approach commonly used for conversational 
analysis. Since I was a member of the group who produced the electronic text, the study would be classified as auto-ethnographic. The object of the study was the ordinary behavior of electronic mail users in the Information Services Division of a large financial institution. As an employee of that Division and as a heavy user of electronic mail, I was able to understand the context of the data that I analyzed. Further, since I had worked for the institution for 23 years and knew all the other participants, I was able to understand the social and psychological background of their communication.

\section{$\underline{\text { Data }}$}

The data used in this study consisted exclusively of electronically produced and transmitted text. I did not interview any of the participants except to obtain their consent to use messages they had created. At the time that they created the messages they were unaware that I would be using them in my study. I made no attempt to determine previous or subsequent conversations, electronic or otherwise.

The context of the electronic mail conversations was the normal daily work environment of the division. It employs about 600 people who work in a very large, twostory operations center in a rural area. The building has large atrium in the center, a cafeteria that is open only at breakfast and lunch, and work areas in which each employee has a private cubicle with five-foot walls. Because of this design, casual face-to-face conversation is limited, with most spoken conversation taking place in pre-planned meetings. All employees have a telephone with Voice Mail and either a computer terminal or personal computer with electronic mail access throughout the corporation. Electronic mail is heavily used because it is more convenient than meetings, provides a record of previous communications, and is connected to a calendar system. It is used for a large variety of functions including work planning, problem solving, management 
reporting, announcements, meeting notices, and personal business of all kinds. There are no management directives about the content or volume of electronic mail messages.

All of the data that I collected for this study was created between April 1993 and May 1994. During this time the corporation was experiencing significant management changes, reorganizations, and cost-cutting programs.

The subjects of the study were computer technicians or mid-level technical managers who had been using electronic mail for at least five years. Since the division has a flat organizational structure, there was very little difference in status among the participants. They represented a wide diversity of educational backgrounds and geographical origins, sharing only a common employer, familiarity with computer technology, and a similar income level. All but about one percent were native English speakers. There were about equal numbers of males and females, with females holding fewer management positions. Ages varied from 25 to 60 with a majority between 35 and 45 .

The electronic mail product used by everyone in the study was IBM's OfficeVision, version 2.1. This product is strictly text-based, with no capability to transmit pictures, color, or sound. Using OV the user constructs a message with a simple text editor, specifies any number of recipients and "copy to" recipients, then sends the message via a central IBM mainframe. The recipients are identified by the sender with an ID consisting of a two-character prefix and a five-digit employee number. My ID, for example, was SS13556, indicating that I was in the Software Services Department with an employee number of 13556 . Each user may create a "nickname file" in which this ID can be associated with a name that is more easily remembered. When the message is sent, both the sender and the intended recipients are identified in the message with their full names as defined in the global user list. The system automatically stores the note in the sender's "note log." 
A "mail waiting" message notifies the recipient that someone has sent a message. After the recipient reads it she can choose any or all of the following options: erase, store, print, reply, forward without a reply, or forward with a reply. This last option is the mechanism with which users can create conversational "chains." A note may be forwarded with a reply any number of times and to any number of recipients, so that each recipient can read the entire preceding conversation. When a "chain" is sent this way the system stores the complete chain in the sender's note log.

When reading a "chain" the user must begin with the last message and read backwards to the first, or most recent, message. Each message is framed with the sender ID, full name, optional sender information such as phone number and job title, and system identifier; the recipient(s) $\mathrm{ID}(\mathrm{s})$, full name(s), and system identifier; a subject heading created by the sender (or "NO SUBJECT" if none is entered); and the date and time. A "chain" of forwarded messages looks like this:

MSG FROM: TR31557 --VMS1 TO: RR29863 --VMS1 03/22/94 10:47:10 To: RR29863 --VMS1 Sarah Smith

FROM: BILL BECKETT

Subject: Schedule

Figured you might .....

*** Forwarding note from RR29863 --VMS1 03/22/94 10:45

To: SP28413 --VMS1 Bill Beckett

FROM: Sarah Smith 372-6685

Subject: Schedule

Good idea.

I would also like to clarify some of the changes you made to the schedule.

*** Forwarding note from TR31557 --VMS1 03/22/94 10:02 ***

To: RR29863 --VMS1 Sarah Smith

FROM: BILL BECKETT

Subject: Schedule

What with the apparent hiring problem perhaps you and I should spend some time to review the Polar Bear schedule? 


\section{Data Collection}

The data used in this study consists of forwarded "chains" that I received on OfficeVision between April, 1993, and April, 1994. As part of my daily routine of reading and replying to my electronic mail, I selected candidate "chains" and stored them in a special note log. My criteria for selecting them was that the "chain" have three or more individual messages and that no single message exceed one screen of text. I did not limit the number of participants. When I was not sure whether to store a candidate, I chose it anyway because I could discard it later. This process yielded a corpus of 69 sets of linked messages or conversations that was stored electronically. I then printed the entire note log. I reviewed this printed log and discarded sets that contained very long individual messages, involved large numbers of participants, or did not otherwise meet my criteria. I eventually chose 16 conversations to be analyzed.

The conversations in the final corpus were printed in reverse order, just as they had appeared to the participants. They also contained their names, employee ID numbers, dates, and times. In order to facilitate analysis, I created a second version of each conversation in which the messages were sequenced in forward temporal order. I reproduced the text exactly as the authors had created it, but I coded their names and employee ID numbers to protect their privacy. If there were internal references to either individuals or corporations, I coded these as well. I preserved gender in coding all names and kept the dates and times on each message. For readability when citing a conversation, I removed the computer system ID and the framing added by the system when the notes were stored and printed.

\section{Privacy and Confidentiality}

The policy on electronic mail in the corporation being studied specified that electronic mail messages are confidential and proprietary to the corporation. Even though 
individuals are free to send messages to anyone inside the corporation, the recipients are not free to share them with outsiders. The policy also states that management must respect individual privacy under normal conditions. Exceptional cases such as fraud, legal action, or employee termination may justify management access to electronic mail that is not their own.

I needed no management approval in order to collect the data since it was all sent to me in the normal course of my work. However, in order to use and publish it for this study, I requested approval from the Information Security Department Manager. His requirements were that I encode all individual and corporate identifiers, get written consent from all the participants, allow contributors whose text was cited in the final report to refuse such use, and submit the final report to him for review before publishing it.

\section{Data Analysis}

To limit the scope of the study I chose four of what I considered to be the most important and interesting characteristics of spoken conversations in relation to their potential manifestations in electronic form. These were openings and closings, the turntaking system, adjacency pairs, and repetition. Even though most of the conversations in my corpus contained data relevant to more than one of these features, I attempted to categorize them for use in a single category. As I examined each category more closely in the analysis phase, I reassigned some of the notes to another category. This became an iterative process until I was satisfied that I had satisfactory data for all the categories.

For each note I wrote a small background summary from my personal knowledge. I then compared it to the features of spoken conversations that I summarized in Chapter II. If there were differences, I attempted to decide if the original feature was missing, modified, or possibly even inappropriate. 


\section{CHAPTER IV}

\section{ANALYSIS OF ELECTRONIC MAIL CONVERSATIONS}

All of the electronic mail "chains" or conversations in the corpus have a number of characteristics in common. They were created by the employees of a single organizational unit in which nearly all the participants knew each other. Some of them have been friends for many years, while others are only acquaintances. They share a common work culture and history along with shared knowledge of the major events that have affected their environment. They are physically located in a single, large building and so have the opportunity to talk face-to-face.

The content of the conversations reflects the daily work and personal interactions of computer professionals. Contrary to possible stereotypes of computer experts, these people have highly varied educational and cultural backgrounds, most having learned about computers on the job. Hence they share a common jargon, use acronyms freely without fear of being misunderstood, but at the same time may use very different language styles. All are native English speakers.

To compensate for the absence of non-verbal devices for communication in electronic mail such as intonation and stress, the participants may invent substitutes. The electronic mail tool that they use severely limits their options since it allows only text and the characters on a standard personal computer keyboard. It is surprising that no one has chosen to draw pictures with parentheses, commas, and other special characters as is common on other electronic mail systems. Physical distance among the participants, a critical factor in regulating face-to-face-conversation, is irrelevant in these electronic conversations. However, the participants are very aware of their place in the 
organizational hierarchy and the social distance that it entails. It is commonly perceived that as the number of hierarchical levels by which the recipient outranks the sender increases, the likelihood of a reply decreases. Most mail is sent either to peers or to members of the organizational level immediately above or below the sender's level.

The standard header in the OfficeVision electronic mail system includes a line labeled "Subject:." If the following blank is not filled, the system supplies "No Subject." Most people, however, supply a subject heading. It may be descriptive ("Business Process Reengineering Class"), obscure ("Suggestion"), a complete message ("Meeting at 1:00 is cancelled"), or even a directive ("Call NANCY at 206-574-3240"). Even when the sender does supply a subject, it may not reflect the real function of her message. An unfortunate feature of the subject heading is that when messages are forwarded multiple times to create a conversational "chain" the heading remains constant. If the topic changes, the subject heading becomes irrelevant.

The particular organizational culture represented in the corpus has a number of taboo subjects. Even though the stated corporate policy is that electronic mail is private, and there are no management directives on acceptable content, people generally understand that they should avoid "unwise" comments. This is partly because the forwarding feature allows the recipient to share messages without informing the sender. Occasionally, a message will end with a request not to forward it. The taboo subjects include overt criticism or ridicule of management, allusions to romantic attachments, politically incorrect references to race, gender, or religion, and mention of illegal activities.

In addition to avoiding the culturally prohibited topics, the participants are generally concerned with maintaining a cooperative work environment. Most tasks in the Division are done by groups, with consensus being the most common method for making decisions. This highly group-oriented environment encourages attention to the sensitivities of others and strongly discourages assertive or demanding language. 


\section{Conversational Openings and Closings}

A conversational speech event can be divided into three parts: the opening, the body, and the closing. The openings and closing are likely, but not required, to be formulaic expressions that occur in pairs. In addition, spoken conversations may begin with a "preopening" (Wardhaugh, 1983) that serves to make the opening possible.

With electronic mail it is not possible to have a "pre-opening" such as eye contact or a smile. The conversation may, however, begin and end with conventional formulaic expressions. Consider the following conversation between two friends, Jack and Sarah, who are negotiating a time to meet outside the office. The subject refers to a Macintosh computer that Sarah has just bought.

\section{[Example 1]}

Subject: Mac/Saturday

Jack: Hi Sarah,

It looks like I will have this Saturday off - - would 9 or 10 am be ok to work on your Mac? I have a new version of the Symbionts Control Panel. I also have the latest virus signature from the Central Point Software for finding an INIT virus that just came out (you might have go a notice about it in the mail from them). I would like to install 'Mac EKG' which will run diagnostics on all of the chips on the motherboard (including Ram!).

See ya, Jack P.

Sarah: Sounds good to me if we can be done by 11:30 - - have to help future daughter-in-law with wedding shopping. We can start at 9 - Will this give us enough time?

Jack: Hi Sarah,

If we start by 9 am there should be plenty of time!

See you then!

Jack $\mathbf{P}$.

$4 / 20 / 94 \quad 16: 55$

Sarah: OK. See ya -- 
Jack begins his first message with the ritualized, "Hi Sarah." His choice of "Hi" over "Hello" suits their relationship as friends. Sarah, however, does not respond to the greeting as she probably would if they were face-to-face. Instead, she begins her reply with a straightforward answer to Jack's question. This pattern repeats in the next two messages with Jack offering his greeting and Sarah not responding. Jack's messages appear to be more like written notes in which it is usual for the greeting to appear at the beginning of each one.

The pattern of closings in this example is more complex. Jack ends both of his messages with a formulaic closing. The first is the casual "See ya" followed by his name. This closing combines a commonly used form from spoken conversation with his name, a form used only in written notes and letters. He includes his last initial possibly because there is more than one Jack in his department and this is his habit. Jack's second closing is the somewhat more formal "See you then!" followed by his name. The exclamation point, however, indicates informality so that the end result seems friendly. Sarah does not respond to Jack's first closing, but she does respond to the second. This is the actual end of the conversation. She confirms the arrangements that have been negotiated with "OK" and then follows with a repetition of Jack's first closing, "See ya." This is the conventional pattern in spoken conversation in which closings are paired exchanges. Her use of the closing from Jack's first message is interesting because it serves to tie the first message in the chain to the last, thus overriding Jack's pattern of structuring each individual message as a complete conversation.

For this conversation we have the overall structure:
A: $\quad$ Greeting 1 Message Closing 1
B: $\quad$ Message
A: $\quad$ Greeting 1 Message 


\section{Closing 2 \\ B: Confirmation + Closing 1}

In the next example Henry is requesting information from Sarah about a procedure. They are colleagues who do not know each other well.

\section{[Example 2]}

Subject: New operator procedure for $\backslash F R B$

Henry: Sarah,

Can you tell me what is the procedure for having a new step added to the LFRB grave shift runsheet?

Specifically, I have a new job that is to run each morning before any of the balance file update jobs are to be started. At present, this job is run at the behest of applications staff, but it is a regular daily procedure, so it should be included as an operational task.

Thanks

Henry

$4 / 6 / 94 \quad 13: 49$

Sarah: I am finding out who that should be and will let you know - -

When do you want the new procedure to start?

$4 / 6 / 94 \quad 17: 23$

Henry: It can start as soon as I've done all the paperwork for it.

\section{Henry}

4/7/94 09:34

Sarah: The procedure is to enter it into the Change System. You need to be sure that Operations is on the notification list. (I think you enter this - )

When the Ops supervisors get the change notification, they will update their run sheets.

Henry has simply used Sarah's name as his opening. Unlike Jack, he does not repeat it in subsequent messages. In spoken conversation a name may be used particularly if the speaker wants to get someone's attention. In the spoken situation, it can serve as both a pre-opening and an opening. Used in an electronic mail message in which the pre-opening is essentially the action of sending the message, the name is not an attention-getting 
device, but simply a greeting. Again, as in the previous conversation, Sarah does not return the greeting. Her reply to Henry's first message is simply a logical contribution to the conversation.

The closing to Henry's first message combines "Thanks" with his name. This differs in structure from spoken conversation in that he is thanking Sarah for the information he requested before she has replied. It also differs in content since one's own name is not used to close a spoken conversation. Sarah does not respond to either the "Thanks" or to the closing as a whole. Henry's second message, although it has no opening, has his name as the closing. At the end of the conversation there is no closing at all, even though it is clear from the content that the conversation is over. Sarah indicates finality by supplying the requested information in statement form. If we exclude consideration of the misplaced "Thanks" the structure of the conversation is:
A: Opening Message Closing
B: $\quad$ Message
Closing
A: $\quad$ Message
B: $\quad$ Message

Some electronic mail conversations, although understandable without additional information, clearly assume a prior conversation. This is the case here in which three friends are confirming arrangements to have lunch away from the office.

\section{[Example 3]}

Subject: Lunch

To: $\quad$ Sarah, Norine 4/06/94 08:55

Kathy: Are we on for lunch today?

To: $\quad$ Kathy, Norine 4/06/94 09:21

Sarah: Today is fine with me 11:30? 
To: $\quad$ Sarah, Norine

4/06/94 09:26

Kathy: 11:30 works for me. Meet by the turnstiles at the front of the building?

To: $\quad$ Kathy, Norine

4/06/94 09:59

Sarah: $\quad$ OK. I'll be there unless someone says otherwise.

Kathy's initial question implies that arrangements have been previously made. She is checking that they are still in effect. She starts directly with her request for confirmation without a greeting. Sarah's brief reply and Kathy's acknowledgment are also without openings or closings. However, Sarah uses "OK" in her final message as a closing. She follows this with an offer to continue the conversation if Norine (who has not contributed to the conversation) does not agree with the arrangements. This inversion of the closing and an offer to entertain further input is unusual in spoken conversation. The structure of this conversation is simple except for the single closing.
A: Message
B: Message
A: Message
B: Closing
Message (to the non-participant)

Finally, there are conversations that begin and end with no obvious opening or closing. The participants in this example have been to a management retreat at a place called Wemme. They enjoyed the discussion group they were in there and are exploring the possibility of continuing it at the office.

\section{[Example 4]}

Subject: "Wemme Group"

Sarah: Do you think we should resurrect this group? With all the changes going on it could prove extremely interesting - - 
I would be glad to work with you to get it started again. Perhaps if you are interested we could chat for about 30 minutes to decide how to proceed.

$4 / 27 / 93 \quad 12: 05$

Les: $\quad$ Yeah, let's do it. . .

I can think of the following; Ted K., Sam C., Bob J., Sarah S., Les A., Mark W., Roger B., Tom B. Do we go into BSD at all? If so, who might be the choice(s), also what about Tim Cole? and Possibly someone from AJM's area.

$4 / 28 / 93 \quad 11: 53$

Sarah: How about we start with the group you suggest and discuss at the first meeting if we want any others?

$4 / 29 / 93 \quad 08: 48$

Les: What about Bob J. actually I think he is now a direct report to Matt Green like Bev and probably would not be in this group?

Sarah: Do you think it really matters that everyone be at the same level? I don't object, but I think we don't need to be inflexible about it.

Although there is no obvious greeting, it is possible to understand the Subject heading, "Wemme Group," as an opening. It serves to recall a shared experience and sets the stage for continuing to talk about it. Sarah refers to it in her first message by calling it "this group." The closing, on the other hand, appears to be truly absent. Sarah may have effectively ended the conversation, however, by using a rhetorical question that contains a veiled criticism. She appears to be telling Les that his concern about including people of different organizational levels is of no consequence. Les' failure to reply could be because he took offence at Sarah's criticism. Another possibility is that he intended to follow Sarah's suggestion that they "chat for about 30 minutes," thus continuing the conversation either on the phone or face-to-face. 


\section{The Turn-taking System}

The turn-taking system described by Sacks, Schegloff, and Jefferson (1974) depends upon turn-constructional units, or the minimal content of a single turn, and transitionrelevance places, or indicators that a new speaker may begin. In electronic mail conversations we would expect to find both of these, but the ability of the speaker to construct a complete turn before sending it varies significantly from the requirement in spoken conversation to construct it while the other participants listen. Since the participants in the conversation cannot witness a turn as it occurs, but rather receive a completed turn, they do not have to anticipate the transition-relevance place. This does not eliminate the need for it, however, since the recipients of a message must determine whether they need to reply. Unless the sender ends with a closing for the entire conversation, this may not always be clear. Wardhaugh's (1983) observation that indicators for ending and starting a turn can be non-verbal suggests that the electronic mail users may have some difficulty with it.

When an electronic mail message is sent to a single individual, confusion about who is being addressed cannot occur as it might in spoken conversation. However, as is frequently the case, when a message is sent to multiple individuals, it is incumbent on the sender to indicate from whom she expects responses. This may differ from spoken conversation in several ways. She may wish to get a response from each individual, thus expecting a violation of the one-speaker-at-a-time rule. Another option is that she wants the person who has an answer for her to reply without knowing in advance which of the addressees it might be. There is also the possibility of requesting a response only if someone disagrees with the message, something that would be a rarity in spoken conversation. The option that the sender has, on thc otier hand, of addressing one or more recipients allows the precedence order of next speaker selection in spoken conversation to apply. If the message is sent to a single individual, the sender in effect 
has selected the next speaker. Speakers may self-select only if she addresses multiple recipients.

One type of transition-relevance place in spoken conversation is a question. Not all questions, however, have the function of selecting the next speaker. Rhetorical questions, for example, can be used for emphasis without indicating that the speaker expects an answer. Both kinds of questions occur in electronic mail conversations. Consider the following exchange between Donna, who is setting up meetings with a consulting firm, and Sarah, whom she is inviting to one of them. Sam is Sarah's department manager.

\section{[Example 5]}

Subject: May 4 with Decisions Consulting - on site audit

Donna: Sarah, Linda Warren of Decisions Consulting would also like to meet with you on May 4. Time available is 15:30 to 16:30 in the TQM conference room (115). I will also invite Sam Collier to stay after his 2:30 - 3:30 meeting for NSC Computers discussion with you.

Donna

Sarah: I'll be there.

Anything I should bring?

Donna: Whatever will help you answer unexpected questions. . . .

Sarah: You mean my BRAIN??

Donna's first message does not end with any obvious indication that Sarah is to reply. Instead, she "signs" her name as if the conversation were completed. Sarah has to decide whether this is the case, or if she should send a response. It would have been interesting to see if Donna would send a second message if Sarah had not replied. This would have followed the turn-taking protocol in which the speaker continues if no one picks up the turn at a transitional relevance place. Sarah does reply, however, with "I'll 
be there," showing that she interpreted Donna's message as an invitation, a type of utterance that normally requires a response. She also may have decided to reply because of her awareness that this meeting was important. She could deduce this from her knowledge that the consultants had been hired by the Divisonal Vice President and from Donna's mention of her manager in the note. Sarah follows her acknowledgment of the invitation with a question that clearly requires a response from Donna. Donna, however, answers indirectly with a statement that indicates she does not know what will be discussed in the meeting. Again, this may have ended the conversation, but Sarah replies with what appears to be a rhetorical question. By capitalizing "brain," and by doubling the question mark, she injects an ironic note that seems to say that if there is nothing she can bring to help her answer questions she will bring her brain as a last resort. This ends the conversation since the question is really not a question, but a commentary on the upcoming meeting. This two-person conversation accomplishes turn-taking first with an implicit message that it is an important invitation, next with an explicit question, and ends with an ironic rhetorical question.

Conversations involving more that two participants offer substantially more complexity in turn-taking than the previous example. The following conversation begins with four participants: the sender, two recipients, and two "observers" who receive copies of the message. The two recipients, Sarah and Steve, report to Bev, the sender. The "observers" are Bev's manager and the manager directly responsible for running the computers. Other participants are added as Sarah tries to respond to Bev's questions. The topic is a recurring problem with an important computer system for which both Bev and Sarah have some responsibility. Bill, the Division Vice President, has embarrassed Bev by asking her about the most recent problem that she had not been told about. Since Sarah reports to her, Bev can demand the information from her. Sarah is as much in the 
dark as Bev so she passes the request on to two people who she thinks might know.

Finally, the lowest person on the totem pole, Mary, has the answer.

\section{[Example 6]}

Subject: NSC Computers queues this weekend

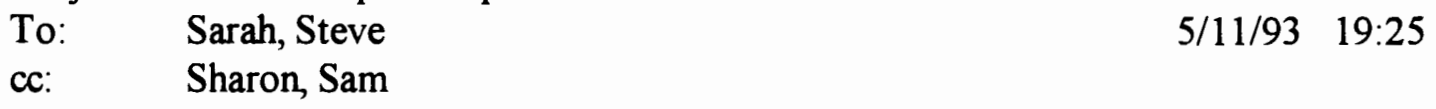

Bev: I hear they (the ubiquitous they) purged the NSC Computer queues this weekend.

a) Did you know?

b) Why did this happen?

c) Who is notified when it happens?

Sarah - would you find out? We need to make this type of info standard knowledge - somehow every time this is necessary we need to know when and why. Bill apparently heard from a customer - again before we told him.

We need to address 'large outage' management and notification as a group.

I also need to know the status of the DEC over the weekend?????

To: Donna, Cindy

$5 / 12 / 93 \quad 11: 48$

Sarah: Can either of you enlighten me on this?

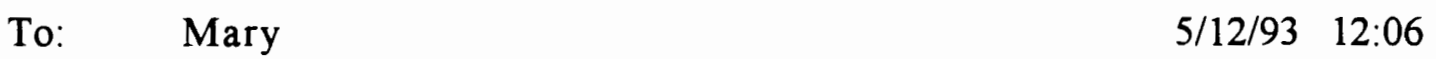

Cindy: Mary, did you know anything about this? I thought I'd ask you since you were not copied on this note. Can you shed any light on this subject?

Thanks!

Cindy

To: $\quad$ Cindy

$5 / 12 / 93 \quad 14: 37$

Mary: I honestly don't know if you forwarded this to me or not. I know there was some mail that I missed. (it's a long story on that). But anyway, my understanding is that the ntos purged the queues either sometime Sunday 
or very early Monday morning because NSC Computers was off host for a good portion of the weekend and the queues were too high.

NSC Computers was off host due to the conversion.

If you want more detailed information, just let me know.

Thanks.

p.s. I haven't forgotten about the minutes from the last weeks NSC Computers working group meeting, just haven't had time to type them up. (but there wasn't much in the way of interest to us). They did discuss the lan going in at USCS, which we will be interested in eventually. Roger Brown spent the last 30 minutes going over the USCS disaster. This was VERY interesting. That was the highlights of last weeks meeting.

To: Donna, Sarah $5 / 12 / 93 \quad 17: 04$

Cindy: I sent this off to Pam, and this is the information that I received from her. Please let me know if I can provide you with anything else. Thank you. Cindy

To: $\quad$ Bev $5 / 13 / 93 \quad 09: 36$

Sarah: (blank)

The string of questions in the opening message appears to be directed to both Steve and Sarah until we reach "Sarah - would you find out?" This marks Sarah for the next turn in the conversation. Bev follows this directive with some explanations and observations. This inversion of the transition relevance place with observations is difficult to imagine in a spoken conversation. We would expect instead that Sarah would pick up her turn immediately after the directive. Next we have a second directive from Bev, "I also need to know the status of the DEC over the weekend?????" Sarah has been working on this system so she knows she is expected to reply. Bev has introduced a new turn constructional unit and a transition relevance place in the same utterance. The overall structure of Bev's message is: 
1. Turn-constructional unit \#1

2. Transition-relevance place \#1

3. Continuation of turn-constructional unit \#1

4. Turn-constructional unit \#2

5. Transition-relevance place \#2

Sarah does not know any of the answers to Bev's questions, so she sends a message to both Donna and Cindy asking for information. She does this with a straightforward question that clearly indicates that either addressee may reply. This message appears to be simply a transition-relevance place with the turn-constructional unit or units from the previous message attached. Such a conversational turn is possible because of the electronic mail feature that attaches all previous messages to the current message. This can also occur in spoken conversation when a participant asks for help in replying to the speaker, but in that case the previous message is held in the participants' memories.

In the next turn Cindy passes the chain to Mary. Her question, "Can you shed any light on this subject," echoes Sarah's question, "Can either of you enlighten me on this?" so that it is clearly tied to the previous turn. Finally, Mary replies to Cindy with the answer. The first part of Mary's note is the answer to Bev's original first question. She turns it back to Cindy with an offer to supply more information if asked. Completely ignoring Bev's second directive, Mary adds her own second turn-constructional unit. Her "p.s." introduces it as a new topic that is added for Cindy's information only. Finally, Cindy replies to both Donna and Sarah with the answers to Bev's first directive. Sarah in turn sends Cindy's answer to Bev without comment. This final "empty" turn uses the attached messages from the other participants as its turn-constructional unit. Sarah does not supply a transition-relevance-place in the form of an offer to get further information or to answer questions. Note that Sarah never answers Bev's question about the DEC. Either Sarah chose to ignore it or she replied using a medium other than electronic mail. 
The process of self-selection can occur in electronic mail conversations as the following example shows. Sarah is trying to find out from a high level manager, Matt, whether he wants to manage a large computer hardware purchase. She sends copies of her message to her manager, Bev, and two individuals who have done this in the past, Bill and Edward.

\section{[Example 7]}

Subject: NSC Computers Hardware Upgrade

To: $\quad$ Matt Green $5 / 21 / 93 \quad 17: 26$

cc: Bev, Bill, Edward

Sarah: I met with Ned Thomas of NSC Computers today to review our requirements. He will have a first cut at a quote next Wednesday.

How would you like to handle it from there? Will someone (you, Edward, me) handle financing options?

Also, shall I be working with A.J. on the management presentation that Bill mentioned in our capacity meeting?

We need to have the production upgrades done by August 1 to support the new ATM software and the Wire Transfer new release, so I'd like to move quickly

Let me know what you'd like me to do.

To: $\quad$ Sarah

Bill Perhaps a first cut at a schedule would be helpful. That way we can see if any "conflicts" exist between these upgrades and the other stuff that Danny Wolfe is working on.

To: Bill $5 / 24 / 93 \quad 15: 13$

Sarah: A schedule would be good - - what is Danny's role in the upgrade?

To: $\quad$ Sarah $5 / 24 / 93 \quad 16: 41$

Bill: His primary focus will be on fixing what's broke. 
To: Bill

Sarah: $\quad$ So what exactly would he DO? Or does he just recommend?

To: $\quad$ Sarah

$5 / 25 / 93 \quad 07: 31$

Bill: We'll have a proposed solution plan to review next week. This should give us a better view of just how much work is ahead. . probably tons

Sarah's first message contains three questions, each of which could have been a transitionrelevance-place in a spoken conversation. None of them function this way here since she follows the third question with an expansion on her initial turn-constructional unit. The actual transition, "Let me know what you'd like me to do," selects Matt, the only direct addressee, as the next speaker. Bill, however, self-selects at this point with a suggestion to Sarah that introduces the issue of a schedule. This is an interruption, normally considered rude in face-to-face interaction, not only because another speaker was clearly selected, but because it changes the focus of the conversation. Bill's message is sent only to Sarah, but does not directly ask for a response. Sarah, however, is confused by the mention of Danny in this context, and so replies to Bill. This message ends with a transition-relevance-place that selects Bill. Bill's reply, "His primary focus will be on fixing what's broke," moves the topic away from the hardware upgrade to another project. It appears that Bill expects this to be the end of the conversation, and so offers no transition to the next speaker. Sarah, however, self-selects by asking Bill about Danny's role. She wants to determine if he will participate in the hardware upgrade or "just recommend." Bill's response ends the conversation by a further comment on his project with no answers to Sarah's original questions. Matt never replies to Sarah's original questions.

This conversation illustrates the local unspoken perception that as the distance up the hierarchy between sender and receiver increases, the likelihood of a reply decreases. 
Even though Sarah's original message clearly selects Matt as the next speaker, he does not reply. This violates the requirement in spoken conversation to respond to questions and requests. When it is ignored the silence itself constitutes a message that the hearer is either arrogant, hostile or opting out of the conversation. In the case of electronic mail conversations, this requirement may be much weaker. Matt's failure to reply may have been intended to send Sarah the message that her questions were either inappropriate or directed to the wrong person.

\section{Adjacency Pairs}

Some conversational turns can be grouped in pairs such that the first turn determines the relevance of the second. Greetings, closings, questions, invitations, challenges, requests, and compliments all commonly occur as pairs. Some pairs, most commonly greetings, closings, and compliments, are formulaic. Others, such as invitations, may be ritualized but offer the possibility of acceptance or rejection. Questions and requests for information offer a large range of possible form and content, including questions paired with questions, requests paired with questions, and requests followed by information. The electronic mail examples discussed above contain adjacency pairs of several types. Example 1 (p. 35) closes with the formulaic pair, "See you then!" followed by "OK. See ya - -." Example 5 (p. 42) begins with an invitation to a meeting that elicits the reply, "I'll be there." This acceptance is immediately followed by another type of pair, a question and answer: "Anything I should bring?" and the reply, "Whatever will help you answer unexpected questions. ...."

The following is an electronic mail conversation consisting of two adjacency pairs. Sarah has invited Bill to talk to a group that she chairs, and wants him to bring some visual aids for his presentation. 


\section{[Example 8]}

Subject: NSC Computer Working Group Talk

$4 / 28 / 93 \quad 12: 24$

Sarah: Would it be possible for you to bring a very basic diagram of the SBS system? (Pictures are easier than words?)

$4 / 28 / 93 \quad 18: 08$

Bill: There are no pictures available yet. I can wing it on a white board?

Sarah: White board would be good - -

The first adjacency pair is Sarah's request, politely put as a question and explained by her parenthesized comment, followed by Bill's statement, "There are no pictures available yet." This appears to be a factual statement, but because of the preceding request it is really a refusal. Bill has softened it by giving the reason he cannot oblige her. Bill initiates the second pair with single utterance that combines a suggestion and a request for approval. Sarah completes the pair by agreeing to his suggestion. In this conversation the pairs do not alternate between the two participants. Rather, Bill supplies both the second part of the first pair and the first part of the second pair. Since his first reply is a refusal, he makes a further contribution to ensure that the conversation will continue. The structure thus is:
A: Suggestion
B: Refusal Suggestion
A: Acceptance

A single message that replies to the previous one and initiates the second one can involve implied acceptance or rejection of a request. Further, adjacency pairs can be interrupted with insertion sequences of one or more adjacency pairs. Both of these occur in the next example in which Sarah and Bill are negotiating a meeting time. 


\section{[Example 9]}

Subject: BOMA Discussion

$$
4 / 4 / 94 \quad 13: 59
$$

Sarah: I will be out tomorrow, but should be here the Tuesdays after that. Anything you need to know for tomorrow's meeting?

4/5/94 07:32

Bill: Not really. Would like to spend some time with you discussing Polar Bear your plans .... etc.

$$
\text { 4/6/94 09:42 }
$$

Sarah: I assume the discussion is not an emergency - - Would you like me to set something up for next week?

Bill: How about this afternoon?

$4 / 6 / 94 \quad 09: 55$

$4 / 6 / 94 \quad 10: 01$

Sarah: $\quad 4: 30 ?$

$4 / 6 / 94 \quad 11: 03$

Bill: $\quad$ Actually .... We could meet at $2: 30$ if you want?

Sarah: I prefer 4:30, if that's OK with you.

$4 / 6 / 94 \quad 11: 05$

$4 / 6 / 94 \quad 11: 05$

Bill: $\quad$ OK ... 4:30.

Sarah's first message and the first two words of Bill's response, "Not really," constitute the first adjacency pair. As in the previous example, he continues the conversation in the same message, this time by making a request to spend "some time." Sarah's reply contains an implied acceptance of his request and a question about implementing his request in a single question, "Would you like me to set something up for next week?" Bill rejects this offer by making an alternative suggestion. This brief message is really the second part of the pair initiated by Sarah and the first part of the next pair. Sarah replies by suggesting a time, a form similar to Bill's previous message in that it completes a previous pair and initiates the next one in a single utterance. Bill interrupts this sequence of paired exchanges by inserting a pair in which he asks to change the time that Sarah has proposed. This insertion sequence (indented in the example) is itself a paired exchange. Sarah rejects his proposal and so the conversation concludes with Bill agreeing to the 
original time. This final message, "OK....4:30," is the response to Sarah's earlier question, "4:30?" The structure of this conversation in terms of adjacencey pairs is:
A: Offer
B: Rejection
A:
Invitation
B: Acceptance
Offer
A: Rejection Suggestion
B: Acceptance Suggestion
A:
B:
Alternate suggestion Rejection
Acceptance

Some forms of adjacency pairs that are found in spoken conversation appear to be absent from the electronic mail conversations collected for this study. "Thanks" occurs several times, but is never followed by the expected, "you're welcome," or similar forms such as "it was nothing." Instead, where gratitude is expressed, it most often occurs before the requested action is completed or information supplied, a structure that requires the hearer to respond with the action or information and not a formulaic response to a thank-you. Thanks may also appear as the closing to a conversation, also without a response. Apologies similarly may lack a response. These structures appear in the following example in which Sarah wants her problem with WORD licenses corrected. Vince is the manager responsible for personal computer software, with Ray reporting to him. Bev is Sarah's manager.

\section{[Example 10]}

Subject: WORD Licenses

To: $\quad$ Vince

cc: $\quad$ Bev

Sarah: Three times in the last two days I have tried to use WORD and gotten the error message indicating all the licenses were being used

What do I have to do to get more purchased?

To: Ray 
Vince: $\quad$ Please check on this \& get back with Sarah

Thanks, Vince

To: $\quad$ Bev

$8 / 05 / 93 \quad 15: 39$

cc: $\quad$ Sarah, Vince

Ray: Sorry about the inconvenience on getting into WORD. We just bought 5 more copies and I increased the count on the LAN this morning

To: $\quad$ Ray

$8 / 06 / 93 \quad 08: 12$

cc: $\quad$ Bev

Sarah: Thanks.

The second message from Vince to Ray requests that Ray check on the situation. It ends with "Thanks" before Ray has had a chance to comply with the request. This "thanks-inadvance" strategy has appeared in two previous examples ( 2 and 6). Ray responds, interestingly, to neither Vince nor Sarah, but to Bev's manager with an apology. One possible explanation for this is that he assumed Sarah's problem prevented her from accomplishing tasks assigned to her by Bev, and that therefore Bev was really the individual who had been inconvenienced. Only Sarah replies to his message, but she completely ignores the apology. Instead, she acknowledges Ray's action to solve her problem and ends the conversation with a simple "Thanks."

In spoken conversation the pairing requirement is so strong that silence in response to the first part of a pair is usually understood to have meaning. In the absence of a legitimate excuse like not having heard the speaker, the other participants will assume that the silence indicates either rudeness, a strong negative reaction to the speaker, or that the hearer is opting out of the conversation. A similar expectation exists with electronic mail, but with the difficulty that it is not possible for the participants to determine if a recipient retrieved a message from the system, i.e., if he "heard" it. In Example 3 (p. 38) in which three women are arranging to meet for lunch, one of the participants, Norine, 
never responds. This may have been because she did not read her mail, or, more likely, because she communicated with one of the others by phone or face-to-face. In this case, there would not be an assumption of rudeness or opting out. We can further assume this because in the final message Sarah makes it explicit that she does not expect a response by stating "I'll be there unless someone says otherwise." Lack of response is also acceptable when the sender asks for information from several participants, expecting a reply only if the recipient can supply it. This was the case in Example 6 (p. 44) in which Sarah asks, "Can either of you enlighten me on this?" In Example 7 (p. 47) the situation is more problematical. Sarah addressed a senior member of the division, Matt, with a direct request, but did not get a response. Because she violated the local protocol that mail not be sent more than one level up the hierarchy, Matt's silence probably represented a rebuke for this unusual behavior. This more closely resembles the perception in spoken conversation that silence is a negative response.

\section{$\underline{\text { Repetition }}$}

Repetition of sounds, words, phrases, and clauses in spoken conversation has several functions. It can be used by the speaker to extend an utterance while she plans what to say next, and it is useful to the hearers as an aid to comprehension. These functions are not needed in electronic mail conversations because the written medium allows planning and comprehension without time pressure. However, repetition can be used to compensate for the lack of features available in spoken discourse like intonation, stress, and body language. In spoken conversation these are important tools for linking parts of a spoken conversation and for foregrounding and emphasis. Repetition can also be used in electronic mail conversations to mark the beginning and conclusion of episodes, to ridicule or trivialize, and to create rapport among the participants. 
Repetition can be an exact echo or an approximate paraphrase or intermediate between the two. Two kinds of repetition are found in the simple conversation cited above (Example 3, p. 38) in which three women are arranging to meet for lunch. The following abbreviated version highlights the exactly repeated words.

Kathy: Are we on for lunch today?

Sarah: Today is fine with me 11:30?

Kathy: 11:30 works for me. Meet by the turnstiles at the front of the building?

Sarah: OK. I'll be there unless someone says otherwise.

"Today" links the first message and the first part of the second as an adjacency pair and "11:30" links the next pair. This not only creates cohesion but serves as a check that the recipient received the sender's message correctly. There is also a repeated structure in this conversation:

Kathy: Are we on for lunch today?

Sarah: Today is fine with me 11:30?

Kathy: 11:30 works for me. Meet by the turnstiles at the front of the building?

Sarah: OK. I'll be there unless someone says otherwise.

Besides having identical meaning, the second phrase mirrors the structure and rhythm of the first. "Is fine" and "works" in this context mean unqualified acceptance, but each takes a different preposition to precede "me." It is interesting that "for" could replace "with" in the first phrase, creating "is fine for me," but the reverse substitution, "works with me," changes the meaning. The two complete phrases could be interchanged in the messages, however, without altering their meaning. This adds to the coherence created with the repeated words, "today" and "11:30," but also contributes to the feeling of rapport in the conversation. By repeating the structure in Sarah's message, Kathy communicates that she not only accepts what Sarah has said, but how she said it.

In Example 4 (p. 39) about the "Wemme Group" we find one phrase repeated both exactly and approximately, and another with structural repetition. 
Sarah: Do you think we should resurrect this group? With all the changes going on it could prove extremely interesting - -

I would be glad to work with you to get it started again. Perhaps if you are interested we could chat for about 30 minutes to decide how to proceed.

Les: $\quad$ Yeah, let's do it. . .

$4 / 27 / 93 \quad 12: 05$

I can think of the following; Ted K., Sam C., Bob J., Sarah S., Les A., choice(s), also what about Tim Cole? and Possibly someone from AJM's area.

$4 / 28 / 93 \quad 11: 53$

Sarah: How about we start with the group you suggest and discuss at the first meeting if we want any others?

$4 / 29 / 93 \quad 08: 48$

Les: What about Bob J. actually I think he is now a direct report to Matt Green like Bev and probably would not be in this group?

$4 / 29 / 93 \quad 11: 54$

Sarah: Do you think it really matters that everyone be at the same level? I don't object, but I think we don't need to be inflexible about it

Les replies to Sarah's "Do you think" with "I can think." In addition, Sarah's last message begins with an exact echo of her opening with "Do you think." In the middle of the conversation we have two suggestions, one beginning with "How about," the other with "What about." These two sets of repeated phrases guide the entire conversation since, with the exception of Les' "Yeah, let's do it. . .," all five messages begin with them.

A true paraphrase, without structural repetition, appears in Example 6 (p. 44). This example also contains two instances of exact repetition and the use of repetition for emphasis.

Subject: NSC Computers queues this weekend

To: $\quad$ Sarah, Steve $5 / 11 / 93 \quad 19: 25$

cc: $\quad$ Sharon, Sam 
Bev: I hear they (the ubiquitous they) purged the NSC Computer queues this weekend.
a) Did you know?
b) Why did this happen?
c) Who is notified when it happens?

Sarah - would you find out? We need to make this type of info standard knowledge - somehow every time this is necessary we need to know when and why. Bill apparently heard from a customer - again before we told him.

We need to address 'large outage' management and notification as a group.

I also need to know the status of the DEC ENGIN over the weekend?????

To: Donna, Cindy $5 / 12 / 93 \quad 11: 48$

Sarah: Can either of you enlighten me on this?

To: $\quad$ Mary $5 / 12 / 93 \quad 12: 06$

Cindy: Mary, did you know anything about this? I thought I'd ask you since you were not copied on this note. Can you shed any light on this subject? Thanks! Cindy

To: Cindy $5 / 12 / 93 \quad 14: 37$

Mary: I honestly don't know if you forwarded this to me or not. I know there was some mail that I missed. (it's a long story on that). But anyway, my understanding is that the ntos purged the queues either soratime Sunday or very early Monday morning because NSC Computers was off host for a good portion of the weelicud and the queues were too high.

NSC Computers was off host due to the conversion.

If you want more detailed information, just let me know.

Thanks. 
(part of the message has been deleted here)

To: Donna, Sarah

$5 / 12 / 93 \quad 17: 04$

Cindy: I sent this off to Pam, and this is the information that I received from her. Please let me know if I can provide you with anything else. Thank you. Cindy

To: $\quad$ Bev

Sarah: (blank)

$5 / 13 / 93 \quad 09: 36$

In the middle of the conversation, Cindy passes Sarah's question, "can either of you enlighten me on this," to Mary, paraphrasing the question with "Can you shed any light on this subject?" In the same message, Cindy repeats Bev's question, "did you know." The other exact repetition, "let me know," may not be an example of repetition for cohesion or emphasis but rather a commonly used phrase that would normally appear in such a conversation.

Bev's repetition and expansion of the word "they" with "the ubiquitous they" in parentheses is clearly an attempt at emphasis with an undertone of criticism. She seems to be saying that she objects to the commonly used third person plural, but is forced to use it because she lacks information. Bev also creates an emphatic message by repeating variations of a clause containing "need to" four times:

We need to make

we need to know

We need to address

I also need to know

"Need to know" is used twice, first with a plural subject and next with the singular followed by "also." This narrowing of the scope of who needs to know contributes to the emphatic tone of the message. The three verbs, "make," "know," and "address," two of which refer to action, support the strong message that she wants not only information, but performance. 
Repetition is used in Example 9 (p. 51) to bound an episode within the larger conversation. In this case repetition marks the adjacency pair around an insertion sequence.

Sarah: I will be out tomorrow, but should be here the Tuesdays after that. Anything you need to know for tomorrow's meeting?

Bill: Not really. Would like to spend some time with you discussing Polar Bear your plans.... etc.

$4 / 6 / 94 \quad 09: 42$

Sarah: I assume the discussion is not an emergency - - Would you like me to set something up for next week?

Bill: How about this afternoon?

$4 / 6 / 94 \quad 09: 55$

$4 / 6 / 94 \quad 10: 01$

Sarah: $\quad 4: 30 ?$

Bill: $\quad$ Actually .... We could meet at 2:30 if you want?

$4 / 6 / 94 \quad 11: 03$

Sarah: I prefer 4:30, if that's OK with you.

$4 / 6 / 94 \quad 11: 05$

$4 / 6 / 94 \quad 11: 05$

Bill: $\quad$ OK $\ldots 4: 30$.

Bill replies to Sarah's suggestion that they meet at 4:30 only after inserting his alternate suggestion. Since Sarah rejects this (by repeating the time that she prefers), he agrees to her earlier message by repeating the time. We can see that emphasis is also achieved here since there can be no doubt at the end of the conversation that they will meet at 4:30.

The use of repetition to express subtle ridicule appears in Example 7 (p. 47).

Subject: NSC Computers Hardware Upgrade

To: $\quad$ Matt Green

$5 / 21 / 93 \quad 17: 26$

cc: Bev, Bill, Edward

Sarah: I met with Ned Thomas of NSC Computers today to review our requirements. He will have a first cut at a quote next Wednesday.

How would you like to handle it from there? Will someone (you, Edward, me) handle financing options? 
Also, shall I be working with A.J. on the management presentation that Bill mentioned in our capacity meeting?

We need to have the production upgrades done by August 1 to support the new ATM software and the Wire Transfer new release, so I'd like to move quickly

Let me know what you'd like me to do.

To: $\quad$ Sarah $5 / 24 / 93 \quad 08: 20$

Bill Perhaps a first cut at a schedule would be helpful. That way we can see if any "conflicts" exist between these upgrades and the other stuff that Danny Wolfe is working on.

To: Bill $5 / 24 / 93 \quad 15: 13$

Sarah: A schedule would be good - - what is Danny's role in the upgrade?

To: $\quad$ Sarah $5 / 24 / 93 \quad 16: 41$

Bill: His primary focus will be on fixing what's broke.

To: Bill $5 / 24 / 93 \quad 17: 47$

Sarah: So what exactly would he DO? Or does he just recommend?

To: $\quad$ Sarah $5 / 25 / 93 \quad 07: 31$

Bill: We'll have a proposed solution plan to review next week. This should give us a better view of just how much work is ahead. . probably tons

Sarah ends her first message by asking for direction on what she should do. Since the person to whom she addressed her request did not reply, and the information she got from Bill was confusing, she paraphrases her original question to apply to another person, Danny. Her following question, "Or does he just recommend," tells us that she does not believe that Danny will do anything to help her, an assumption that is supported by her emphasis, by using capitals, on the word "do." Sarah thus indirectly 
ridicules people who only recommend and at the same time expresses her frustration at not receiving an answer to her question. 


\section{CHAPTER V}

\section{RESULTS}

Analysis of the electronic mail messages cited in the previous chapter revealed both similarities to and differences from spoken conversation. Openings and closings, turn taking, adjacency pairs, and repetition all occurred in the data, but all had some differences from spoken interaction. The degree of similarity or difference varied, however, among the structural features studied.

\section{Conversational Openings and Closings}

In spoken conversation there may be a pre-opening in the form of a smile or eyecontact. This functions as a way to make it possible to start the conversation to follow. In electronic mail exchanges a pre-opening of this kind can not occur because there is no option for non-verbal communication. However, in one of the cases examined (Example 4) the subject header, "Wemme Group," may have functioned as a pre-opening. It referred to an experience that the sender and addressee had recently shared and so set the stage for the message to follow.

Some of the electronic mail exchanges began with greetings of the same form as in spoken conversation such as "Hi." Others began with the name of the addressee, a form unusual in spoken conversation except as a pre-opening or attention getting device. In some cases the greeting was repeated at the beginning of each message in the conversation, resembling the form used in personal letters. In no case did the recipient reply to an initial greeting as is obligatory when people are face-to-face. Many of the exchanges lacked openings entirely. 
Conversational closings followed some of the patterns found in openings, but there were also new patterns. Closings were often absent, but when they were used, they either resembled spoken closings or included the sender's name. Like openings, some appeared in the middle of a conversation as an ending to an individual message. Differing from openings, which never occurred as pairs, some closings were typical paired exchanges like "See you then" and the reply, "See ya." In one conversation the final message ended with the formulaic closing, "OK," followed by an offer to entertain further input "if someone says otherwise" (Example 3). This would be very unusual in spoken interaction. Another closing strategy that would rarely occur in spoken conversation was an expression of gratitude in advance for what had been requested. This use of "thanks" as a closing was sometimes followed by the sender's name as if the message were a letter. In some of the cases in which no closing occurred it was not possible to tell whether the conversation had ended or if it was continued using another medium such as phone or face-to-face interaction.

\section{The Turn-taking System}

As in spoken conversation, both turn-constructional units and transition-relevance places occurred. Some exchanges closely resembled spoken conversation with brief individual messages and clear transition-relevance places. However, in others there were several important modifications to the turn-taking system. In one case (Example 4), a single message contained two complete turn-constructional units and two transitionrelevance places. In another (Example 6), one message contained a second turnconstructional unit following the closing. In both cases, the senders introduced a new topic with the second conversational unit.

Non-verbal cues play an important role in the turn-taking system, particularly in the process for selecting the next speaker. Lacking these, there was considerable confusion in 
some of the electronic mail exchanges about whether a reply was expected and from whom it should come. Even though electronic mail recipients do not have to anticipate transition-relevance places, they still must determine whether they need to reply to a message. In electronic conversations involving only two participants, there is no problem in the selection process, but it can be difficult to determine when the conversation has ended. This was the case in Example 4. When there are multiple participants the sender may identify the next sender, as in Example 6. Here the precedence order found in spoken conversation would apply so that no one else would self-select after the speaker has made her selection. In Example 7 the sender clearly selected the next sender by addressing the message to a single person, while sending only copies to others. However in this conversation, one of the "observers" self-selects even though he received only a copy. This violates the precedence order for turn-taking, but unlike spoken conversation, the interruption by the observer is not considered to be rude. The original addressee in this example never replied which sent a less objectionable message than would silence in a spoken conversation.

Other anomalies in the selection process for the next sender also appeared in the data. In one case the sender expected simultaneous responses from several recipients (Example 3), violating the one-person-speaks-at-a-time rule, while in another (Example 6) the sender requested a reply from anyone who could answer her question. This "does anyone know" strategy can be used in spoken interaction, but it is particularly convenient with electronic mail. In Example 3 the last message contained a request for a reply only if someone disagreed with the arrangements made in the conversation. This would also be unusual, though possible, in spoken conversation. In Example 6 one of the messages consisted only of a transition-relevance place with the attached preceding message forming the turn-constructional unit. In the same example the final message is blank so 
that the attached message again forms the conversational unit, but the lack of a transitionrelevance place indicates the end of the conversation.

The other devices used to indicate a transition-relevance place were generally the same as in spoken conversation. Questions were the most common form used, and a rhetorical question (Example 5) was not confused as a transition-relevance place. Also, similar to spoken interaction, there were observers in the form of recipients of message copies, and participants were added to and dropped from ongoing conversations.

\section{Adjacency Pairs}

Openings and closings are two of the most common types of formulaic adjacency pairs found in spoken conversation. In the electronic conversations studied, openings never occurred as pairs while closings occurred both singly and as pairs. Questions commonly occurred followed either by an answer or another question. In one example (9) a question and answer pair was separated by another question and answer pair or an insertion sequence. Invitations and requests were most often followed by either acceptance or rejection. In one case (Example 10), a request was followed by a reply, but the reply was sent to the requester's manager rather that to the requester. This kind of pairing would not occur in a spoken conversation.

In addition to openings, there were other utterances that normally occur in pairs in spoken interaction, but did not in the electronic conversations. There is an apology in Example 5 that does not elicit a response. In the same conversation, a "Thanks" is not paired with a reply such as "You're welcome." In Example 6 a message that is sent to two people is paired with a response from only one. It is not clear whether this violates the pairing requirement for spoken conversation because of the unusual selection process for the next speaker. Here a lack of response, considered rude in face-to-face interaction, is 
acceptable for at least one of the participants. Elsewhere, however, (Example 3) ignoring the pairing requirement after a question does send a negative message.

\section{$\underline{\text { Repetition }}$}

Repetition appeared in all the electronic conversations and was used to accomplish most of the same functions as it does in spoken conversation. In addition, it was used to compensate for the lack of intonation, stress, and body language. In spoken conversation these are important for indicating emphasis and for creating rapport. The forms of repetition found in the data varied from exact duplication to a rough paraphrase. In Example 3 there were both kinds. In this example, the repetition served to create coherence and to reinforce the rapport between the two participants. Repetition was used in Example 1 to mark the beginning and end of the conversation, and in Example 9 it marked the beginning and end of an episode within the conversation. In Example 6 the use of multiple repetitions, with variations in the person of the subject and in the verb, but not in the form, served to emphasize that the sender expected action in response to her message. Mild ridicule in Example 7 was accomplished with a paraphrase of a question to which the sender did not receive an answer.

In spoken conversation the speaker has to be sure that the other participants comprehend what she is saying. Repetition is one of the techniques available for this purpose. Since with electronic mail "chains" the participants can read all the earlier utterances, it would seem that this use of repetition would not appear. However, some of the senders used repetition to reinforce their message. In Example 9 a meeting time appeared three times, and in Example 3 the time and day to meet for lunch appeared twice. 


\section{$\underline{\text { Summary }}$}

Of the structural features studied, repetition was used in ways most similar to its uses in spoken conversation. The feature having the most differences from spoken interaction was the turn-taking system. There was more variation in the sender's selection of the next sender, including the option for multiple simultaneous replies. Also prominent among the differences from spoken conversation was the tendency of a sender to continue her message after a transition-relevance place. Openings, closings, and other adjacency pairs found in spoken conversation were much less likely to be complete pairs. Openings, apologies, and thanks most commonly lacked replies.

These findings are based on the assumption that the features of spoken conversation that I analyzed would be manifested in the text of the electronic mail messages. There is, however, the possibility that some structural elements can be marked by features that do not appear in the text itself. For example, notification by the mail system that there is a message waiting to be read could function as a pre-opening to an electronic conversation. In the case of an "empty" message used to pass on other attached messages it would be possible to attribute content to the "empty" message even in the absence of text. The intended communicative function was accomplished by using the "forward" feature of the mail system rather than with an explicit text message. Also outside the message text itself were exchanges in which the lack of a response functioned as either a neutral or negative response, and the interpretation depends on knowledge of the larger context. Were another researcher to include consideration of elements such as these that exist outside the text of the electronic mail messages, she might find more similarities between electronic mail exchanges and spoken conversation than I was able to identify. 


\section{CHAPTER VI}

\section{CONCLUSIONS AND DIRECTIONS FOR FUTURE RESEARCH}

In spite of the small number of electronic exchanges examined in this study, there are several factors that suggest a wider application of the findings. A brief review of a larger sample from the same source indicates that the features identified occur commonly in other electronic mail conversations. They are clearly typical within the organization that was studied, and it is not unreasonable to assume that they may occur in other environments as well.

\section{Conclusions}

A clear difference between the electronic mail conversations studied and spoken conversation is that there is less pressure for brevity in electronic mail. In spoken conversation the speaker must actively keep speaking or another participant may interrupt, but with electronic mail the sender may construct her message with time to reflect and expand upon it. The tendency of a sender to say too much, that is, to add to an already completed message, is one result of this. When the sender is aware that another chance to contribute to the conversation is not directly under her control, she may be tempted to elaborate her message or even begin a new topic. Balancing this, however, in an organization that uses electronic mail extensively, is the time constraint upon the user to reply to a large number of messages in a short period of time. This is a new kind of time constraint, but a real one in the organization studied.

A second characteristic of electronic mail conversations is that the familiar turn-taking system used in spoken conversation has limited usefulness for selecting the next sender. 
This is partly due to the larger number of options that the sender has to address multiple recipients and to send copies to others. A contributing factor may be that senders assume the rules have not changed, and so do not adequately compensate for the lack of clear transition-relevance places in their electronic conversations. A related difficulty is the frequent lack of a conversational closing in an electronic mail exchange so that the recipient does not know if she should reply. This is complicated by the availability of other communication channels such as phone and face-to-face interaction. An electronic mail conversation may be part of a larger conversation carried out using multiple communication channels.

Although the impact of varying time intervals between messages in a single electronic exchange was not addressed directly, it is probable that it affects the structure of the conversation. Those electronic exchanges in which the time intervals were small tended to resemble spoken conversation more closely, having shorter utterances and more repetition across messages. It is possible that participants in such exchanges were able to complete these conversations without interruption from other electronic mail messages or communication with another medium. When an individual must answer multiple messages quickly, the switching among conversations can be expected to create some loss of cohesion and rapport among the participants.

Adjacency pairs, common in spoken conversation, are not consistently used in electronic mail. The lack of replies to openings, the "thanks in advance" structure, and the absence of a reply to an apology may all be due to the nature of the medium. These strategies all serve to streamline the electronic conversation rather than appearing rude or uncooperative. Here again is an effect of time pressure on the sender that comes from a source other than the possibility of being interrupted as in spoken conversation.

The tendency of some senders to organize their messages more as letters or memos than as contributions to a conversation is an indication that the conventions used in 
electronic mail are still evolving. Some users appear to transfer what they do in spoken conversation to their electronic mail exchanges while others transfer their habits from written communication.

\section{Applications}

An awareness of the ways that electronic mail conversations differ from spoken conversation can be useful in several ways. Given the difficulties described above in applying the turn-taking system, particularly for selecting the next sender, users of the medium may be able to modify their messages accordingly. Electronic mail users who are frustrated by these differences, but who do not understand them, may react inappropriately. Some individuals in the corporation that I studied avoid electronic mail in favor of spoken conversation even when it is less efficient. Others have published guidelines that attempt to solve the difficulties. These are interesting in that the divisional change management team has recently addressed some of the problems found in this study. Among their "E-Mail Communication Protocol" guidelines are:

1. No need for thank you's, you can thank "in advance."

2. No need for return confirmation notes, unless a response is requested.

3. If a note is sent to more than two people and an answer is needed, indicate who you expect to get the response from, otherwise each recipient might wait for someone else to respond.

4. Don't use E-mail when you could accomplish what you need in a face to face, or phone call.

The first two guidelines acknowledge that thanks "in advance" and return confirmation notes do indeed occur. The third is an attempt to correct the next sender selection difficulty. The last suggests that face-to-face conversation might be better, perhaps because its rules are better known. The tendency for some electronic mail senders to say 
too much is also discussed in these guidelines with the recommendation that long messages include a summary at the top of the note in bold or capital letters with the text to follow.

In designing new electronic mail systems or in evaluating existing systems it is important to be clear about the functions that it will serve and the ways in which its users will accomplish these functions. Some of the data in this study suggests that individuals will use electronic mail as a substitute for spoken conversation, while others will use it as a tool for transmitting information in memo form. Design features can be introduced to enhance either approach, and possibly may accommodate both.

As one form of ethnographic study, an analysis of the forms and functions of electronic mail conversations can contribute to an understanding of the particular organizational culture in which it is used. New employees might consider such a study in order to increase their effectiveness in the organization. Managers would certainly gain useful insights into the unspoken assumptions and values that operate in the areas for which they are responsible.

\section{Directions for Future Research}

Investigation of the structure of electronic mail conversations is clearly an area for fruitful research since so little has been done so far. Most of the studies to date have addressed either the functions of electronic mail systems or the use of electronic mail in relation to other channels of communication. Further, the medium is so new that both the medium itself and the conventions for its use are still evolving. The researcher who explores this domain is certain to find new linguistic features and structures emerging from this evolution.

The current study was limited to a particular organization operating in a particular time and place. A single electronic mail tool was used, and only four structural features of 
spoken conversation were analyzed. This immediately suggests avenues for future study. Electronic mail conversations may vary by the type of organization using it. Academic usage and business usage could be compared. A new opportunity for studying emerging organizational uses of this tool will soon be available when the Portland Fire Bureau installs electronic mail terminals in its fire engines and rescue vehicles. In this particular case it would be possible to collect data on how novices used the system and how their usage changed over time. Other contextual factors that may affect electronic mail usage are gender, age, education, and social status.

The electronic mail tool itself probably affects the linguistic structures that its users choose. For example, bulletin boards that allow multiple participants to have simultaneous access to the messages sent by others would necessarily use a different turn-taking system from the one in this study. Also, availability of pictures or sound would expand and probably alter the ways in which conversational coherence and participant involvement are achieved.

Many features of spoken interaction that might appear in electronic mail exchanges were not studied here. Phatic communication, spoken versus written style, framing of the conversation, contextualization cues, techniques for maintaining cooperation, and syntactical structures are only some of the areas remaining to be investigated. In addition, it would be useful to know how much of the features studied can be accounted for by the medium and how much is due to the style of the organization or of the individuals. Is the variation attributable to factors such as degree of involvement or detachment of the participants the same kind of variation that we find in spoken conversation? Further, does the structure of the conversation vary less than the style?

Communication styles may also differ according to the functions being performed. Most of the conversations in this study were concerned with getting information or arranging meetings. A few functioned to initiate an action related to the work 
environment. Among the functions not studied were social maintenance, problem solving, political advancement, personal communication, and entertainment. An extended study of either the same organization or of others may reveal additional functions as well as differing linguistic structures. 


\section{REFERENCES}

Allerton, D. J. (1991). The greater precision of spoken language: Four examples from English. English Studies: A journal of English language and literature. 72(5), 470478.

Blackman, B. I. (1990). A naturalistic study of computer-mediated communication: Emergent communication patterns in online electronic messaging systems. (Doctoral dissertation, The Florida State University, 1990.) (From Dissertation Abstracts International, 51(3), 51-03A, p. 678.)

Brown, R. \& Ford, M. (1961). Address in American English. Journal of abnormal and social psychology. 62, 375-85. (also in J. Laver \& S. Hutcheson (Eds.), Communication in face-to-face interaction (pp. 128-145). Baltimore: Penguin.)

Chafe, W. L. (1982). Integration and involvement in speaking, writing, and oral literature. In D. Tannen (Ed.), Spoken and written language: Exploring orality and literacy (pp. 35-53). Norwood, NJ: Ablex.

Clark, H. H., \& Schaefer, E. F. (1989). Contributing to discourse. Cognitive Science, $\underline{13}$, 259-294.

Feldman, M. S. (1987). Electronic mail and weak ties in organizations. Office: Technology and People, 3, 83-101.

Fish, R. S., Kraut, R. E., \& Chalfonte, B. L. (1990). The VideoWindow system in informal communications. In Proceedings of the Conference on ComputerSupported Cooperative Work, October 7-10, 1990, Los Angeles, CA (pp. 1-11).

Ford, C. E. (1993). Grammar in interaction: Adverbial clauses in American English conversations. Cambridge: Cambridge University Press.

Grice, H. P.(1975). Logic and conversation. In P. Cole \& J. L. Morgan (Eds.), Syntax and semantics, Volume 3, Speech acts. New York: Academic Press. 
Goffman, E. (1955). On face-work: An analysis of ritual elements in social interaction. Psychiatry, 18, 213-31. (also in J. Laver \& S. Hutcheson (Eds.), Communication in face-to-face interaction (pp. 274-288). Baltimore: Penguin.)

Hatch, E. (1992). Discourse and language education. Cambridge: Cambridge University Press.

Hall, E. T. (1964). Silent assumptions in social communication. In D. McK. Rioch \& E. A. Weinstein (Eds.), Disorders of communication. Research Publications, Association for Research in Nervous and Mental Diseases, $\underline{42}, 41-55$. (also in J. Laver \& S. Hutcheson (Eds.), Communication in face-to-face interaction (pp. 274288). Baltimore: Penguin.)

Hopper, R. (1983). Interpretation as coherence production. In Craig, T., \& Tracy, K. (Eds.). (1983). Conversational coherence: Form, structure, and strategy (pp. 8198). Beverly Hills: Sage.

Labov, W. (1972). Rules for ritual insults. In Sudnow, D. (Ed.). (1972). Studies in social interaction (pp. 120-169). New York: The Free Press.

Malinowski, B. (1923). The problem of meaning in primitive languages. In supplement to Ogden, C., \& Richards, I. The meaning of meaning. Routledge \& Kegan Paul. (also in J. Laver \& S. Hutcheson (Eds.), Communication in face-to-face interaction (pp. 146-152). Baltimore: Penguin.

Murray, D. E. (1991). Conversation for action: The computer terminal as medium of communication. Philadelphia: John Benjammins.

Pomerantz, A. (1978). Compliment responses: Notes on the co-operation of multiple constraints. In Schenkein, J. (Ed.). (1978). Studies in the organization of conversational interaction (pp. 79-112). New York: Academic Press.

Porter, P. (1984). "Dear PP05" Interpersonal communication and the computer: Entering a brave new world. Library Hi Tech, 2(1), 23-27. 
Reder, S., \& Schwab, R. G. (1989). The communicative economy of the workgroup: Multi-channel genres of communication. Office: Technology and People, 4 , 177195.

Richards, J., Platt, J., \& Weber, H. (Eds.). (1985). Longman dictionary of applied linguistics. Essex: Longman.

Sacks, H., Schegloff, A., \& Jefferson, G. (1974). A simplest systematics for the organization of turn-taking for conversation. Language, $\underline{50}$ (4), 696-735. (A variant version is in Schenkein, J. (Ed.). (1978). Studies in the organization of conversational interaction (pp. 7-55). New York: Academic Press.)

Saffo, P. (1994). Hot new medium: Text. Wired, 1, (2), America Online, 3-13-94. Sanders, R. E. (1983). Tools for cohering discourse and their strategic utilization: Markers of structural connections and meaning relations. In Craig, T., \& Tracy, K. (Eds.). (1983). Conversational coherence: Form, structure, and strategy (pp. 67-80). Beverly Hills: Sage.

Schegloff, E. A. (1972). Notes on a conversational practice: Formulating place. In Sudnow, D. (Ed.). (1972). Studies in social interaction (pp. 75-119). New York: The Free Press.

Schenkein, J. (1978). Sketch of an analytic mentality for the study of conversational interaction. In Schenkein, J. (Ed.). (1978). Studies in the organization of conversational interaction (pp. 1-6). New York: Academic Press.

Sproull L., \& Kiesler, S. (1991). Computers, networks, and work. Scientific American, 265, 116-123.

Sproull, L., \& Kiesler, S. (1986). Reducing social context cues: Electronic mail in organizational communication. Management Science, 32, 1492-1512. Tannen, D. (1989). Talking voices. Cambridge: Cambridge University Press 
Tannen, D. (1990). You just don't understand: Women and men in conversation. New York: Ballantine.

Wardhaugh, R. (1985). How conversation works. Oxford: Basil Blackwell. 
APPENDIX

ELECTRONIC MAIL CORPUS 


\section{Conversation 1}

Subject: Mac//Saturday

Jack: Hi Sarah,

It looks like I will have this Saturday off - - would 9 or 10 am be ok to work on your Mac? I have a new version of the Symbionts Control Panel. I also have the iatest virus signature from the Central Point Software for finding an INIT virus that just came out (you might have go a notice about it in the mail from them). I would like to install 'Mac EKG' which will run diagnostics on all of the chips on the motherboard (including Ram!).

See ya, Jack P.

$4 / 20 / 94 \quad 14: 34$

Sarah: $\quad$ Sounds good to me if we can be done by 11:30 - - have to help future daughter-in-law with wedding shopping. We can start at 9 - -

Will this give us enough time?

Jack: Hi Sarah,

If we start by $9 \mathrm{am}$ there should be plenty of time!

See you then!

Jack P.

$4 / 20 / 9416: 55$

Sarah: OK. See ya --

Situation: Sarah has bought a new computer system for her home and is having a colleague help with installing software. 


\section{Conversation 2}

Subject: New operator procedure for $\mathrm{FRB}$

$4 / 6 / 94 \quad 12: 27$

Henry: Sarah,

Can you tell me what is the procedure for having a new step added to the IFRB grave shift runsheet?

Specifically, I have a new job that is to run each morning before any of the balance file update jobs are to be started. At present, this job is run at the behest of applications staff, but it is a regular daily procedure, so it should be included as an operational task.

Thanks

Henry

$4 / 6 / 94 \quad 13: 49$

Sarah: I am finding out who that should be and will let you know - -

When do you want the new procedure to start?

$4 / 6 / 94 \quad 17: 23$

Henry: It can start as soon as I've done all the paperwork for it.

Henry

4/7/94 09:34

Sarah: The procedure is to enter it into the Change System. You need to be sure that Operations is on the notification list. (I think you enter this - )

When the Ops supervisors get the change notification, they will update their run sheets.

Situation: Sarah represents Operations to other departments. Henry is a software developer who needs to get some documentation for Operations changed. 


\section{Conversation 3}

To: $\quad$ Sarah, Norine

Subject: Lunch

Kathy: Are we on for lunch today?

4/06/94 08:55

To: Kathy, Norine

Sarah: Today is fine with me 11:30?

4/06/94 09:21

To: $\quad$ Sarah, Norine

4/06/94 09:26

Kathy: 11:30 works for me. Meet by the turnstiles at the front of the building?

To: Kathy, Norine

Sarah: OK. I'll be there unless someone says otherwise.

4/06/94 09:59

Situation: Three colleagues are meeting for a lunch outside the building. 


\section{Conversation 4}

Subject: "Wemme Group"

$4 / 19 / 93 \quad 20: 20$

Sarah: Do you think we should resurrect this group? With all the changes going on it could prove extremely interesting - -

I would be glad to work with you to get it started again. Perhaps if you are interested we could chat for about 30 minutes to decide how to proceed.

$4 / 27 / 93 \quad 12: 05$

Les: $\quad$ Yeah, let's do it. . .

I can think of the following; Ted K., Sam C., Bob J., Sarah S., Les A., Mark W., Roger B., Tom B. Do we go into BSD at all? If so, who might be the choice(s), also what about Tim Cole? and Possibly someone from AJM's area.

$4 / 28 / 93 \quad 11: 53$

Sarah: How about we start with the group you suggest and discuss at the first meeting if we want any others?

$4 / 29 / 93 \quad 08: 48$

Les: What about Bob J. actually I think he is now a direct report to Matt Green like Bev and probably would not be in this group?

Sarah: Do you think it really matters that everyone be at the same level? I don't object, but I think we don't need to be inflexible about it.

Situation: A discussion group had started at a management retreat. Two participants are discussing whether to continue the group at the office. 


\section{Conversation 5}

Subject: May 4 with Decisions Consulting - on site audit

4/20/94 13:10

Donna: Sarah, Linda Warren of Decisions Consulting would also like to meet with you on May 4. Time available is 15:30 to 16:30 in the TQM conference room (115). I will also invite Sam Collier to stay after his 2:30 - 3:30 meeting for NSC Computers discussion with you.

Donna

Sarah: I'll be there.

$4 / 22 / 94 \quad 15: 19$

Anything I should bring?

$4 / 22 / 94 \quad 15: 55$

Donna: Whatever will help you answer unexpected questions. . . .

$4 / 22 / 94 \quad 16: 00$

Sarah: You mean my BRAIN??

Situation: In Donna's first message, the "also" indicates that she expects Sarah has already heard about the meeting. 


\section{Conversation 6}

Subject: NSC Computers queues this weekend

To: Sarah, Steve

$5 / 11 / 93 \quad 19: 25$

cc: Sharon, Sam

Bev: I hear they (the ubiquitous they) purged the NSC Computer queues this weekend.

a) Did you know?

b) Why did this happen?

c) Who is notified when it happens?

Sarah - would you find out? We need to make this type of info standard knowledge - somehow every time this is necessary we need to know when and why. Bill apparently heard from a customer - again before we told him.

We need to address 'large outage' management and notification as a group.

I also need to know the status of the DEC over the weekend?????

To: Donna, Cindy

$5 / 12 / 93 \quad 11: 48$

Sarah: Can either of you enlighten me on this?

To: Mary $5 / 12 / 93 \quad 12: 06$

Cindy: Mary, did you know anything about this? I thought l'd ask you since you were not copied on this note. Can you shed any light on this subject?

Thanks!

Cindy

To: Cindy

Mary: I honestly don't know if you forwarded this to me or not. I know there was some mail that I missed. (it's a long story on that). But anyway, my understanding is that the ntos purged the queues either sometime Sunday or very early Monday morning because NSC Computers was off host for a good portion of the weekend and the queues were too high. 
NSC Computers was off host due to the conversion.

If you want more detailed information, just let me know.

Thanks.

p.s. I haven't forgotten about the minutes from the last weeks NSC

Computers working group meeting, just haven't had time to type them up. (but there wasn't much in the way of interest to us). They did discuss the lan going in at USCS, which we will be interested in eventually. Roger Brown spent the last 30 minutes going over the USCS disaster. This was VERY interesting. That was the highlights of last weeks meeting.

To: Donna, Sarah $5 / 12 / 93 \quad 17: 04$

Cindy:I sent this off to Pam, and this is the information that I received from her. Please let me know if I can provide you with anything else. Thank you. Cindy

To: Bev $5 / 13 / 93 \quad 09: 36$

Sarah: (blank)

Situation: Bill, Bev's boss, has embarrassed her by asking her about a problem that she had not been told about. Since Sarah reports to her she demands the information from her. Sarah is as much in the dark as Bev so she passes the request on to two people who she thinks might know. Finally, the lowest person on the totem pole, Mary, has the answer. 


\section{Conversation 7}

Subject: NSC Computers Hardware Upgrade

To: $\quad$ Matt Green

$5 / 21 / 93 \quad 17: 26$

cc: Bev, Bill, Edward

Sarah: I met with Ned Thomas of NSC Computers today to review our requirements. He will have a first cut at a quote next Wednesday.

How would you like to handle it from there? Will someone (you, Edward, me) handle financing options?

Also, shall I be working with A.J. on the management presentation that Bill mentioned in our capacity meeting?

We need to have the production upgrades done by August 1 to support the new ATM software and the Wire Transfer new release, so I'd like to move quickly

Let me know what you'd like me to do.

To: $\quad$ Sarah

$5 / 24 / 93 \quad 08: 20$

Bill Perhaps a first cut at a schedule would be helpful. That way we can see if any "conflicts" exist between these upgrades and the other stuff that Danny Wolfe is working on.

To: Bill

Sarah: A schedule would be good - - what is Danny's role in the upgrade?

To: $\quad$ Sarah

$5 / 24 / 93 \quad 15: 13$

Bill: $\quad$ His primary focus will be on fixing what's broke.

To: Bill

$5 / 24 / 93 \quad 17: 47$

Sarah: So what exactly would he DO? Or does he just recommend?

To: $\quad$ Sarah

$5 / 25 / 93 \quad 07: 31$ 
Bill: We'll have a proposed solution plan to review next week. This should give us a better view of just how much work is ahead. . probably tons.

Situation: Sarah is asking Matt Green how he wants to handle a large computer hardware purchase. He is the new manager of the Operations area and Sarah is checking on whether the previous way of handling this has changed. Danny is a consultant who is working with Bill on organizational and infrastructure issues, so Sarah is surprised that he might have anything to do with the hardware purchase. 
Conversation 8

Subject: NSC Computer Working Group Talk

$4 / 28 / 93 \quad 12: 24$

Sarah: Would it be possible for you to bring a very basic diagram of the SBS system? (Pictures are easier than words?)

$4 / 28 / 93 \quad 18: 08$

Bill: $\quad$ There are no pictures available yet. I can wing it on a white board?

$4 / 28 / 93 \quad 18: 17$

Sarah: White board would be good - -

Situation: Sarah has invited Bill to talk to a group that she chairs. She wants him to bring some visual aids for his presentation. 


\section{Conversation 9}

Subject: BOMA Discussion

$4 / 4 / 94 \quad 13: 59$

Sarah: I will be out tomorrow, but should be here the Tuesdays after that. Anything you need to know for tomorrow's meeting?

4/5/94 07:32

Bill: $\quad$ Not really. Would like to spend some time with you discussing Polar Bear . . your plans .... etc.

4/6/94 09:42

Sarah: I assume the discussion is not an emergency - - Would you like me to set something up for next week?

Bill: How about this afternoon?

4/6/94 09:55

$4 / 6 / 94 \quad 10: 01$

Sarah: $\quad 4: 30 ?$

$4 / 6 / 94 \quad 11: 03$

Bill: $\quad$ Actually .... We could meet at 2:30 if you want?

$4 / 6 / 94 \quad 11: 05$

Sarah: I prefer 4:30, if that's OK with you.

$4 / 6 / 94 \quad 11: 05$

Bill: $\quad$ OK . . 4:30.

Situation: Sarah normally attends Bill's weekly status meetings on projects that they share. Sarah's project is called Polar Bear, and Bill wants to know how it may affect his projects. They report to different managers, so they negotiate as equals. 


\section{Conversation 10}

Subject: WORD Licenses

To: $\quad$ Vince

$8 / 05 / 93 \quad 15: 13$

cc" Bev

Sarah: Three times in the last two days I have tried to use WORD and gotten the error message indicating all the licenses were being used.

What do I have to do to get more purchased?

To: $\quad$ Ray

$8 / 05 / 93 \quad 15: 39$

Vince: Please check on this \& get back with Sarah

Thanks, Vince

To: $\quad$ Bev

cc: $\quad$ Sarah, Vince

$8 / 05 / 93 \quad 15: 39$

Ray: Sorry about the inconvenience on getting into WORD. We just bought 5 more copies and I increased the count on the LAN this morning

To: Ray

8/06/93 08:12

cc: $\quad$ Bev

Sarah: Thanks.

Situation: The word processing products are available on a shared LAN server. Vince manages the department responsible for this software. Sarah is frustrated that she couldn't use it. Bev is her boss; Vince is Ray's boss. 


\section{Conversation 11}

Subject: Time Capsule, etc.

Cindy: Yes, Sarah, I would be willing to work on this a bit. I do know that Morris still has a box that I made up for him while on the project, that was for 'Time Capsule' stuff. There's an old phone, some photographs, etc. . . in there right now. I think the idea kind of died because know one really wanted the responsibility of it and wasn't sure what to actually do with it. Let's put our heads together and maybe invite Morris to coffee and think of something creative. I'd be willing to help!

PS - I think the garden plots turned out great, they really add to the building! We've already been successful with one of our ideas, why not try more?!! Cindy

Sarah: Great! How about a cafeteria lunch next Wednesday?

Cindy: Sarah, sorry it's taken me awhile to respond to this. Wednesday of next week won't work for me. How about Friday the 30th? Let me know if this will work for you.

4/24/93 09:59

Sarah: Like around 1:30?

$4 / 27 / 93 \quad 08: 30$

Cindy: Friday for lunch at 1:30 sounds great to me. I will meet you upstairs at $1: 30 \mathrm{pm}$. Have a good day! Cindy

$4 / 27 / 93 \quad 10: 48$

Sarah: OK. See you there.

Situation: In a previous discussion Sarah suggested that Cindy help her set up a company museum. Cindy had worked on the project to move the Division to a new building and so has some items saved from the old one. The two women had previously worked together to make small plots available to employees for planting flowers. 


\section{Conversation 12}

Subject: NSC Computers Multi-Session Capability

To: $\quad$ Sarah

$4 / 30 / 94 \quad 11: 40$

Shirley: A couple weeks ago a vendor demo'd a multi-session product. Since then discussion came up that this may not be the way to go to meet this need. I have a task on my list to review vendor documentation in light of security. I am not going to review this documentation until I know there is some real possibility of considering the product.

Please keep me informed on the status of this situation so I know if I need to read-up.

Thanks, Shirley

To: $\quad$ Shirley

$4 / 30 / 9412: 41$

cc: $\quad$ Norine, Don

Sarah: OK. Norine and Don are working on the first part of the business case - what we need it for, etc.

To: $\quad$ Sarah $4 / 30 / 9412: 51$

Shirley: Does this mean we are going ahead with the product that was demo'd

To: $\quad$ Shirley

$4 / 30 / 94 \quad 12: 53$

Sarah: If Ops wants it, it is their call. BSD really doesn't get to say - - except we are always interested in getting input.

Situation: A person outside the decision-making group has been asked by her manager to review a software product that another department wants to buy. Sarah is the spokesperson for the decision-making group. 


\section{Conversation 13}

Subject: Conference Room 116

To: Mike Johnson

Carol: I need a large conference room with a phone for a meeting from 1 to $5 \mathrm{pm}$ on Wednesday, August 4th. Room 115 is the one room I've been able to find that only has one meeting that would need to be re-scheduled - which is your meeting

Would you be able to find another room for your meeting on this date? I would really appreciate it!

Thanks! Carol Ann Herman - Group 4 - CRISP System

To: $\quad$ Carol Ann Herman

$7 / 26 / 94 \quad 08: 42$

Mike: That's not my meeting, I only scheduled it. The meeting is Beverly French's. I'm sure something can be worked out or our meeting cancelled. Please check with Beverly.

To: $\quad$ Beverly French

$7 / 26 / 4 \quad 11: 59$

Carol: Is there any way you could meet in a room other than CONF116 on August 4th? I have a large group and will be teleconferencing people in for a 1 to 5:00 meeting that day, and 116 looks like my best bet. Please let me know - Carol Ann

To: $\quad$ Carol Ann Herman $7 / 26 / 94 \quad 12: 47$

Beverly: It's fine with me if we move the meeting. Mike, will you let everyone know where we are? Thanks.

To: Beverly French, Dennis W. Smith, Toni Anderson, Laura J. Henderson, Dick Swann, Pat Harris, Ann Montgomery

Mike: I don't think I missed anyone, but if I did, forward it along. Our KSP meeting on August 4 has been moved to Conference Room 118.

Situation: Carol Ann needs a conference room for a long meeting, but none are available. The person who reserved the room she wants, Beverly, is two management levels above Carol Ann. 


\section{Conversation 14}

Subject: DEC H/W Maintenance Contract

To: $\quad$ Les

$4 / 20 / 94 \quad 11: 21$

cc: Bev, Laura

Brad: I have a pressing need to add and old DEC printer to our DEC maintenance contract, but I don't know who is currently managing our contract these days. The printer is a LG02 s/n\# NU81805. It is located on PL-6 in the Trust Operations area. Site contact is Laura Halliday, 542-3764.

Then I want to request an immediate $\mathrm{P} / \mathrm{M}$ on the unit to help resolve an open trouble ticket, (\# 638009).

Thanks for your immediate attention in advance.

BH

To: Ben

$4 / 22 / 93 \quad 09: 35$

cc: $\quad$ Bev, Sam

Les: Any suggestions on how this is to be done?

To: $\quad$ Sarah $4 / 25 / 93 \quad 07: 36$

Ben: Sarah---How was this done in the past??

To: $\quad$ Ben $4 / 25 / 93 \quad 09: 08$

cc: $\quad$ Debbie, Bev, Les, Sam

Sarah: Debbie Olson had an agreement with DEC. When she found equipment that was not on their maintenance contract, she would notify them and they would add it. I think it was a simple process - - from our point of view at least.

Debbie could probably tell you who she worked with at DEC.

To: Les

cc: $\quad$ Bev, Laura

Brad: Has there been any direction for adding a printer to the DEC contract? We have a printer down, and customer service is affected. Thanks again. BH 
To: $\quad$ Ben

4/26/93 08:09

cc: $\quad$ Brad, Sarah

Bev: $\quad$ I know you saw the notes from Sarah --- David Dorris was handling Debbie's backup work-- how/where do you want Brad's q. handled?

Situation: Brad is responsible for repairs to printers, but does not negotiate maintenance contracts. Sarah's department used to do it, but the function has moved to Ben's area. Because Ben is new at his job, he goes back to Sarah for information. 


\section{Conversation 15}

Subject: Printer Problems

To: $\quad$ Sarah, Debbie

$6 / 14 / 93 \quad 10: 27$

Julia: I will be out at the OPS center for a meeting at 1:00 today and could meet with anyone who would like after that to discuss printers again. I have feedback from a couple of end-users on how things have been going since they sent in their list of problems.

I cannot find Bev Lee's number in the Corporate Directory (in All-in-1) and I thought if there was a chance I could meet her, that would be nice. I didn't know who she was until Ellen Gray Lewis explained it all to me. (Did I even get the name right?)

To: $\quad$ Bev

$6 / 14 / 93 \quad 10: 33$

Sarah ? ?

To: $\quad$ Sarah

$6 / 16 / 93 \quad 16: 45$

Bev: so why am I not in the corporate directory you use????? I feel slighted. (or is it Holmes)

To: $\quad$ Bev

$6 / 17 / 93 \quad 12: 08$

Sarah: Warren is the mail bridge guru - - I've asked him to investigate.

Situation: Sarah and Bev have responsibility for providing computer services to Julia. Bev has just been assigned this job, but Sarah has been doing it for some time. Julia wants to meet the new person so she can get a direct response to her problems. Bev has recently been married and uses the new name of Holmes. Hence Julia cannot find her in the corporate directory. 


\section{Conversation 16}

Subject: Suggestion

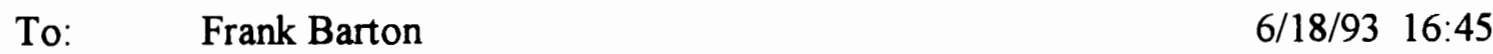

Sarah: (I hope I haven't already sent this idea to you - - if I have just do PF4.)

For your document on Service Level Agreements you could put a Question and Answer section at the end for detail that would muddy up the main text - like:

Q. What about down time because of a $\mathrm{D} / \mathrm{R}$ test?

A. Etc., etc., - - whatever it is.

To: $\quad$ Sarah

$6 / 18 / 93 \quad 16: 48$

Frank: Sarah: I'd rather not put a $Q$ \& A section in the document, but I would like to address any questions which we come up with. As I hear questions, I will address them in the body of the document. I hope this works for you ...

To: $\quad$ Frank

$6 / 21 / 93 \quad 09: 33$

Sarah: Ted and I discussed this a little this morning. We both felt that too much text would discourage people from reading and using it. Which brought up the question of who the audience is and what the purpose is.

Of course this is your document - - only making suggestions.

To: $\quad$ Sarah

$6 / 21 / 93 \quad 11: 08$

Frank: Linda: Off the top of my head: the audience is all $\mathrm{U} / \mathrm{S}$ staff and any interested customers, and the purpose is to give an overview of what an SLA is and how to develop and use one. Hope this helps...

To: $\quad$ Ted

$6 / 21 / 93 \quad 11: 09$

Sarah: Comments?

Situation: Frank has initiated a new process to create Service Level Agreements between providers and customers. Sarah wants to support him and has been discussing his documentation with her colleagues. 